PALEO

Revue d'archéologie préhistorique

$18 \mid 2006$

Varia

\title{
Les Fieux : une occupation gravettienne du Causse quercinois
}

Les Fieux: a Gravettian occupation of the Quercy Causse

Patricia Guillermin

\section{(2) OpenEdition}

Journals

Édition électronique

URL : http://journals.openedition.org/paleo/167

DOI : $10.4000 /$ paleo.167

ISSN : 2101-0420

Éditeur

SAMRA

\section{Édition imprimée}

Date de publication : 1 décembre 2006

Pagination : 69-94

ISSN : $1145-3370$

\section{Référence électronique}

Patricia Guillermin, «Les Fieux : une occupation gravettienne du Causse quercinois », PALEO [En ligne], 18 | 2006, mis en ligne le 23 avril 2009, consulté le 07 juillet 2020. URL : http:// journals.openedition.org/paleo/167 ; DOI : https://doi.org/10.4000/paleo.167

\section{(c) $($ ) $(9)$}

PALEO est mis à disposition selon les termes de la licence Creative Commons Attribution - Pas d'Utilisation Commerciale - Pas de Modification 4.0 International. 


\title{
LES FIEUX : \\ UNE OCCUPATION GRAVETTIENNE DU CAUSSE QUERCINOIS
}

\author{
Patricia GUILLERMIN ${ }^{(1)}$
}

\begin{abstract}
Résumé : Le matériel lithique de la couche E du gisement des Fieux avait été attribué à l'Epipaléolithique puis au Protomagdalénien par F. Champagne qui fouilla le site de 1967 à 1991. La présence de pointes et micropointes de la Gravette ainsi que des burins du Raysse permet, de fait, de rattacher cet ensemble au Gravettien. Cependant, la question de son originalité demeure. Celle-ci ne peut être interprétée comme la conséquence d'un processus taphonomique, l'étude technologique et typologique appuyant l'hypothèse d'homogénéité de la majeure partie de l'ensemble. Par ailleurs, la forte proportion d'armatures, envisagée comme une conséquence de la spécialisation du site dans l'activité cynégétique, met en avant le facteur fonctionnel dans cette interprétation. Celui-ci est en mesure de répondre partiellement à la question de l'association peu commune de différents types d'outils dans l'ensemble de la couche E. D'une part, les lamelles à dos tronquées, nombreuses au sein d'un ensemble où les pointes et micropointes de la Gravette sont bien représentées, témoignent d'une stratégie d'exploitation des ressources siliceuses très adaptée à l'environnement, optimisée pour répondre à l'objectif d'une occupation spécialisée. D'autre part, d'après les travaux de L. Klaric sur le Gravettien moyen à burins du Raysse dans la partie septentrionale de la France, l'interprétation des assemblages du Sud-Ouest de la France associant des éléments rayssiens à ceux d'un système de production d'armatures à dos, doit être discutée. Ainsi, les trois prismes d'interprétation du polymorphisme du technocomplexe gravettien sont toujours d'actualité, regroupant la chronologie, le régionalisme et la fonction des sites.
\end{abstract}

Mots-Clés : Quercy, Gravettien, technologie lithique, polymorphisme, spécialisation, stratégie économique.

Key-words : Quercy, Gravettian, flint technology, polymorphism, specialization, economic strategy.

\section{Abridged english version \\ LES FIEUX : A GRAVETTIAN OCCUPATION OF THE QUERCY CAUSSE.}

The site of "Les Fieux" is located on the causse of Gramat and was excavated by F. Champagne from 1967 till 1991 (fig. 1). The central area of the site consists of a dip created by the collapsing of a karstic gallery situated bellow. Several porches at both eastern and westemextremities at the same level proove that the gallery network had some extension. (fig. 2).

The stratigraphy of this site is shown in various manner accordingly to each area. It expresses some occupation evidences over a wide chronological period of time from mousterian till neolithic period (fig. 3).

Within this stratigraphy, the layer E comes out due to its volume and the eccentricity of its content. It consist of a thick layer of silt covering cryoclastics deposits. Fernand Champagne interpreted this phenomenon as an evidence of a post-würmien climatic change dating from the "Epipaléolithic" (fig. 4). Based on this assumption it was later interpreted as corresponding to the final Gravettian industries. The recent re-study of the material revealed the presence of Gravette's points and micro points (fig. 5) associated to their standard operative procedure followed to obtain their support, so described as "bipolar" (fig. 13), as well as the "burins du Raysse" (fig. 6). All these elements allow the connection of the layer $\mathrm{E}$ to the Gravettian period.

Unfortunately, the stratigraphic data for the various area of the site cannot be exploited to support this connection further. On the Western area, the gravettian evidences are very weak and no specific layer was yet individualised. Nevertheless, one shall mention the presence of one point of "Font-Robert", and several points of "Gravette" as well as some "burins du Raysse" shall be noticed. (fig. 10). On the East, F. Champagne presents a stratigraphy mapping where the main Gravettian area, layer E, with the Solutrean layer (F1a), would be overlapping two Gravettian layer (F1b and F1c) and one Aurignacian layer (F2) (fig.7). One of the two first Gravettian layer, F1c, was dated from $23900 \pm 330$ BP (Gif 6303). Nevertheless, these two places represent a weak number of findings (Around fifty tools) and their profile was not determined precisely. Also, the F1a layer gathers some Gravettian elements, and some Solutrean tools that were found during the excavation extension towards the eastern porch in an area where the $\mathrm{E}$ layer is not present anymore (fig. 8). This finding forces to reconsider the idea that the $E$ layer was above one solutrean layer. The truth is that the entire stratigrafic mapping needs to be reconsidered. In this new study, one shall definitively limit ourselves to the Central area where the lithical remainings clearly corners one Archeological layer (fig.9 et 11). 
The determination of this peculiar assemble, is mainly based on the typo-technologic study of the material.

The high proportion of projectile points, viewed as a consequence of the specialization of the site in cynegetic activities, emphasizes the functional factor in this interpretation. The latter can answer partially the question posed by the uncommon association of different types of tools in the collection of layer E. On one hand, the backed truncated bladelets are numerous within a collection where Gravette's points and micropoints de la Gravette are well represented, and reveal a strategy to exploit mineral resources which is very adapted to the environnement, optimized to reach the objective of a specialized occupation.

These were mainly produced with a very specific and local flint, Jaspéroïds Infralias (fig.15). The structure of this raw material has a thiner grain fitting better to the production of thin and sharp projectile points. This material quality seems to be seeked mostly for lateral projectile points. The carver followed a converging unipolar procedure fitting both to the production of sharp tools, and to a raw material with a thin grain (fig.14). This observation reveals a strong technical investment highly related to the hunting that was performed around the site. The preferences in the exploitation of various flint resources, demonstrate the economical group strategy that seems to have one important impact in the assembly building (fig.15).

On the other hand, L. Klaric proposes a septentrional model of the "Gravettien moyen à burins du Raysse". That opens the discussion on the interpretation of the collections from South-West of France, which combine rayssiens elements to those of a production system of backed pointes. Thus, the three prisms of the gravettian technocomplex polymorphism interpretation are of topicality, gathering the chronology, the regionalism and the function of the site.

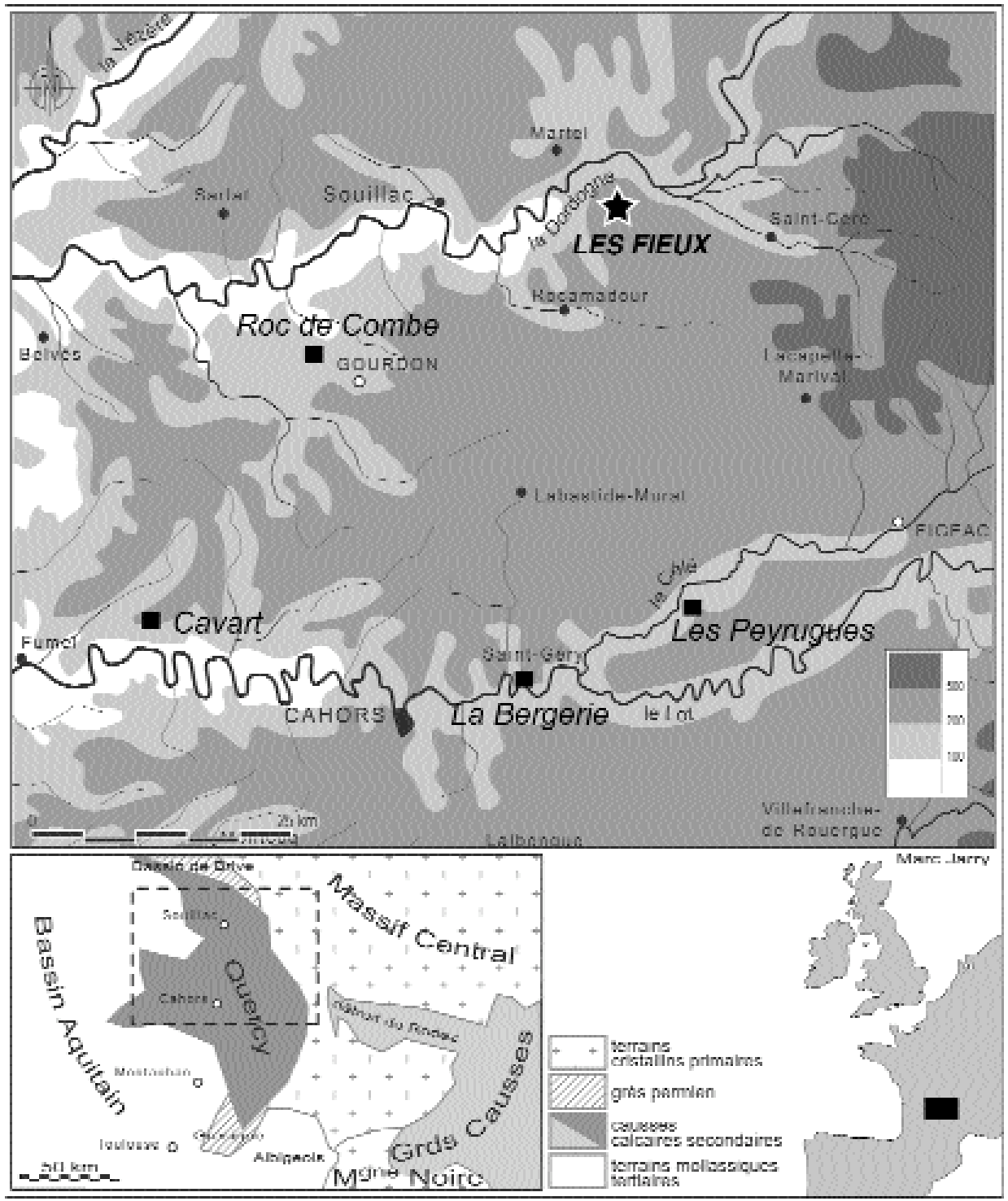

Figure 1 - contexte géographique et géologique des principaux gisements gravettiens du Quercy [...].

Figure 1 - Geographical, and geological presentation of the main gravettian sites in Quercy. [...]. 


\section{INTRODUCTION}

Le Quercy est longtemps resté le parent pauvre de son célèbre voisin, le Périgord. Aujourd'hui, l'intérêt des préhistoriens se tourne davantage vers cette région, notamment dans le cadre d'une démarche pluridisciplinaire menée par une Action Collective de Recherche, ACR (M. Jamy dir). En dehors d'indices d'occupations issus de fouilles anciennes ou de ramassages de surface, les gisements gravettiens sont peu nombreux en Quercy (fig. 1) mais peuvent présenter des caractères intéressants pour l'appréhension des modalités d'exploitation de cette région et de son insertion au sein des territoires paléolithiques. L'exemple présenté ici est le gisement des Fieux, situé sur le causse de Gramat. ; F. Champagne, qui dirigea les fouilles sur ce site pendant plus de 20 ans, y distingua plusieurs niveaux gravettiens stratifiés. Le plus récent, correspondant à la couche $\mathrm{E}$, est aussi le plus important. L'originalité de cet ensemble, qui lui valut une première attribution à l'Épipaléolithique, pose encore aujourd'hui le problème de sa signification chrono-culturelle précise. Son étude permet d'aborder, à nouveau, la question de l'interprétation du polymorphisme des industries gravettiennes. Cette réflexion constitue le fil directeur du travail mené sur les modalités d'exploitation et d'occupation du territoire en Quercy par les groupes du Gravettien (Guillermin 2005).

\section{1- LA COUCHE E DANS SON CONTEXTE ENVIRON- NEMENTAL ET HISTORIQUE}

\section{1- Présentation du gisement}

\subsection{1- Une occupation du causse}

Les paysages du Haut-Quercy sont marqués par la présence des causses, vastes plateaux calcaires du Jurassique moyen et supérieur dont les limites abruptes dessinent des vallées encaissées.

C'est dans ce paysage que se trouve le gisement des Fieux, sur la partie la plus septentrionale du causse de Gramat, à trois kilomètres au nord-ouest de la commune de Miers (fig.1).

La situation de ce gisement, sur un point culminant du plateau, le distingue des sites en grottes et abris creusés dans les falaises bordant les vallées de la Dordogne, du Lot et de leurs affluents. Situé à la confluence de deux vallées sèches se rejoignant pour aboutir 7,5 kilomètres plus loin, à la Dordogne, le gisement n'est pourtant pas à l'écart de l'axe de circulation que devait constituer cette vallée pour les groupes préhistoriques. En outre, l'approvisionnement en eau était vraisemblablement possible du fait de l'activité karstique du causse (Champagne et al. 1996). Cette activité est à l'origine de la formation de nombreuses gale-
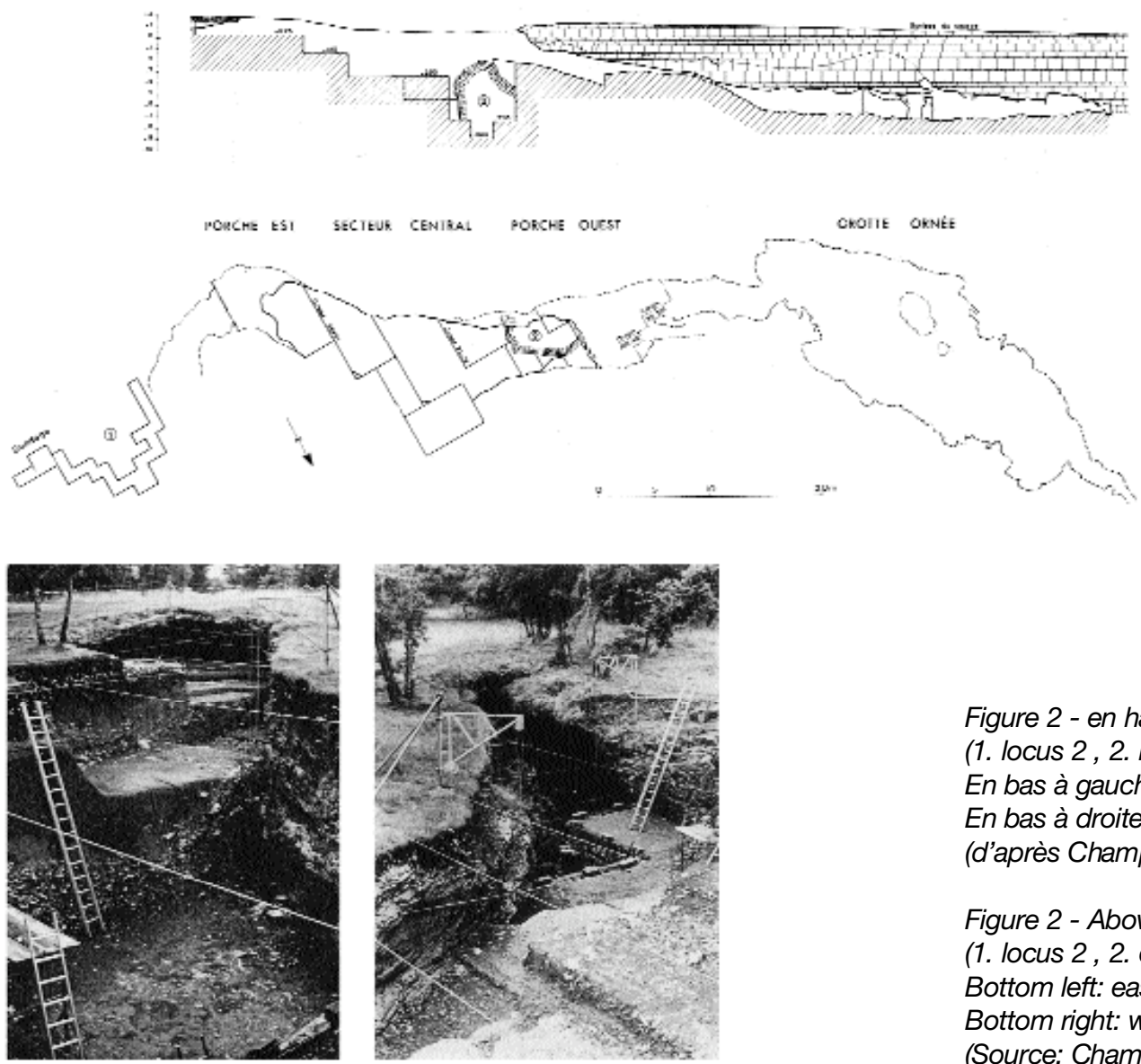

Figure 2 - en haut : plan général du gisement (1. locus 2 , 2. massif stalagmitique effondré) En bas à gauche : porche est En bas à droite : porche ouest (d'après Champagne et al. 1990).

Figure 2 - Above: Complete site layout (1. locus 2, 2. collapsed stalagmitic massif Bottom left: eastern porch Bottom right: western porch (Source: Champagne et al. 1990). 
ries " sous-cutanées " formant un large réseau à l'intérieur des causses. L'effondrement partiel d'une de ces galeries, la reliant à la surface, constitue le gisement des Fieux.

\subsection{2 - Historique des travaux}

Une équipe du spéléo-club de Bergerac découvre la gro tte ornée des Fieux en 1964. Le gisement proprement dit fut découvert en 1966 par le propriétaire, monsieur Caminade, alors qu'il exécutait des travaux de dégagement du porche à l'entrée de la grotte.

Le site est ensuite fouillé de 1967 à 1991 (Champagne 1977 et 1986-1987 ; Champagne et Jaubert 1979 ; Champagne et al. 1990 et 1996 ; Champagne 1996). Les campagnes sont menées sous la direction de F. Champagne et R. Espitalié jusqu'en 1975. Par la suite, F. Champagne dirigera seul les opérations avec la collaboration de J. Jaubert de 1976 à 1985 ainsi que celle d'A. Fournier.

Le gisement est divisé en trois principaux secteurs. Dans un premier temps, les fouilles ont concerné le porche à l'entrée de la grotte (porche ouest). Elles ont, par la suite, été étendues vers l'extérieur, à l'est, découvrant le secteur effondré du boyau karstique. À cette zone effondrée succède un nouveau porche (le porche est) ouvrant sur la poursuite du karst dont l'étendue du réseau reste indéterminée. Un sondage a néanmoins été effectué plus à l'est derrière le porche, mettant en évidence un second effondrement la galerie. Ce sondage correspond au locus 2 tandis que le reste du gisement représente le locus 1 (fig. 2). À terme, les fouilles ont entièrement délimité la partie effondrée de la galerie dont l'ouverture, " au niveau du sol rocheux, [...] mesure $30 \mathrm{~m}$ de longueur sur une largeur maximale de $9 \mathrm{~m}$, avec une orientation générale nordouest/sud-est" (Champagne et al. 1990, p.2).

Les témoignages artistiques découverts aux Fieux furent étudiés parallèlement par plusieurs spécialistes (Glory 1965 ; Nougier 1965a et b ; Lorblanchet 1984a et b). Jusqu'à présent, le lien entre ces témoins et les couches archéologiques n'a pas été clairement établi.

\section{2 - Le problème de l'attribution chronologique de la couche $\mathrm{E}$ ou l'historique d'un raisonnement scientifique.}

Les caractères originaux de la couche $E$ sont établis d'une part par le remplissage auquel elle correspond et d'autre part par l'ensemble lithique qu'elle contient. À 'instar de nombreux préhistoriens, F. Champagne a corrélé l'étude du matériel à l'interprétation chrono-climatique du remplissage sédimentaire de ce puissant niveau (Belounis, 1987).

\subsection{1 - La couche $\mathrm{E}$ : un témoin de changement climatique ?}

Nous reprenons ici les hypothèses avancées par F. Champagne pour la formation du site. Celle-ci s'organiserait en cinq grandes phases :

1. Formation du karst et sédimentation souteraine.

2. Ouverture du karst puis chutes de blocs et de plaquettes consécutives à l'ouverture du karst.
3. Formations cryoclastiques issues de l'effritement des parais et des vestiges de la voûte. Dans le secteur central, ces formations ont cessé dès que les parois ont été colmatées en totalité, mais elles se sont prolongées jusqu'à la fin du Würm dans le secteur est. L'épaisseur des dépôts cryoclastiques (plus de $10 \mathrm{~m}$ ) n'est pas surprenante si on prend en compte simultanément la fragilité de la roche encaissante (calcaire jurassique moyen) et la durée de la séquence stratigraphique [ces formations renferment les niveaux moustériens, aurignaciens et gravettiens].

4. Mise en place d'un dépôt limoneux d'origine éolienne qui comble entièrement le chenal formé par les dépôts cryoclastiques [c'est la couche E].

5. Sédiments post-würmiens (contemporains du Sauveterrien) puis sédiments plus récents (probablement médiévaux) qui colmatent définitivement les deux porches.

A ces grandes phases, il convient d'ajouter divers phénomènes annexes : érosions, colluvionnement et ruissellement par exemple " (Champagne et al. 1990, p.2).

F. Champagne met en avant une nette distinction entre les couches cryoclastiques et le remplissage de la couche $E$ qui le surmonte : cette demière est composée d'un important dépôt de limon argileux très homogène, de couleur brunrouge foncé et totalement dépourvu d'éléments calcaires grossiers. Ce dépôt, dont la puissance est par endroit supérieure à trois mètres, comble la dépression - qualifiée de " chenal " formée par les couches sous-jacentes.

L'hypothèse retenue pour l'origine de sa formation est celle d'une sédimentation d'origine éolienne (Belounis 1987). L'os n'étant pas conservé, aucune datation n'a été réalisée pour le niveau archéologique présent à l'intérieur de cette puissante couche.

1.2.2 - Une étude conditionnée par l'interprétation chronoclimatique du remplissage.

L'interprétation de l'ensemble de la couche $E$ a posé des problèmes à $F$. Champagne ainsi qu'à J. Jaubert, lorsque ce dernier l'a étudié dans le cadre d'un travail de maîtrise en 1979 (Jaubert 1979).

F. Champagne avait distingué des stratigraphies différentes suivant les secteurs (fig. 3). Ainsi, la couche E est au-dessus d'un niveau aurignacien dans le secteur central et au-dessus d'indices gravettiens voire d'un ensemble attribué au Solutréen dans le secteur est (nous discuterons plus loin de la réalité de cette observation stratigraphique). Elle se trouve, d'autre part, sous des occupations sauveterriennes et néolithiques. Le niveau F1c livre une date à 23900 $\pm 330 \mathrm{BP}$ (Gif 6304) tandis que les niveaux sauveterriens (du porche ouest) ont livré comme date la plus ancienne 9450 \pm 190 BP (Gif 1807). Cela réserve un grand intervalle chronologique dans lequel la couche E peut se placer.

F. Champagne s'est fondé sur l'interprétation chrono-climatique du remplissage afin de ressemer cet intervalle. $\mathrm{Ce}$ raisonnement a tenu une place très importante au sein de la communauté scientifique : les préhistoriens l'utilisent pour comparer et interpréter en terme de contemporanéité ou de succession les stratigraphies des différents sites (Laville et Rigaud 1973). Cette démarche 


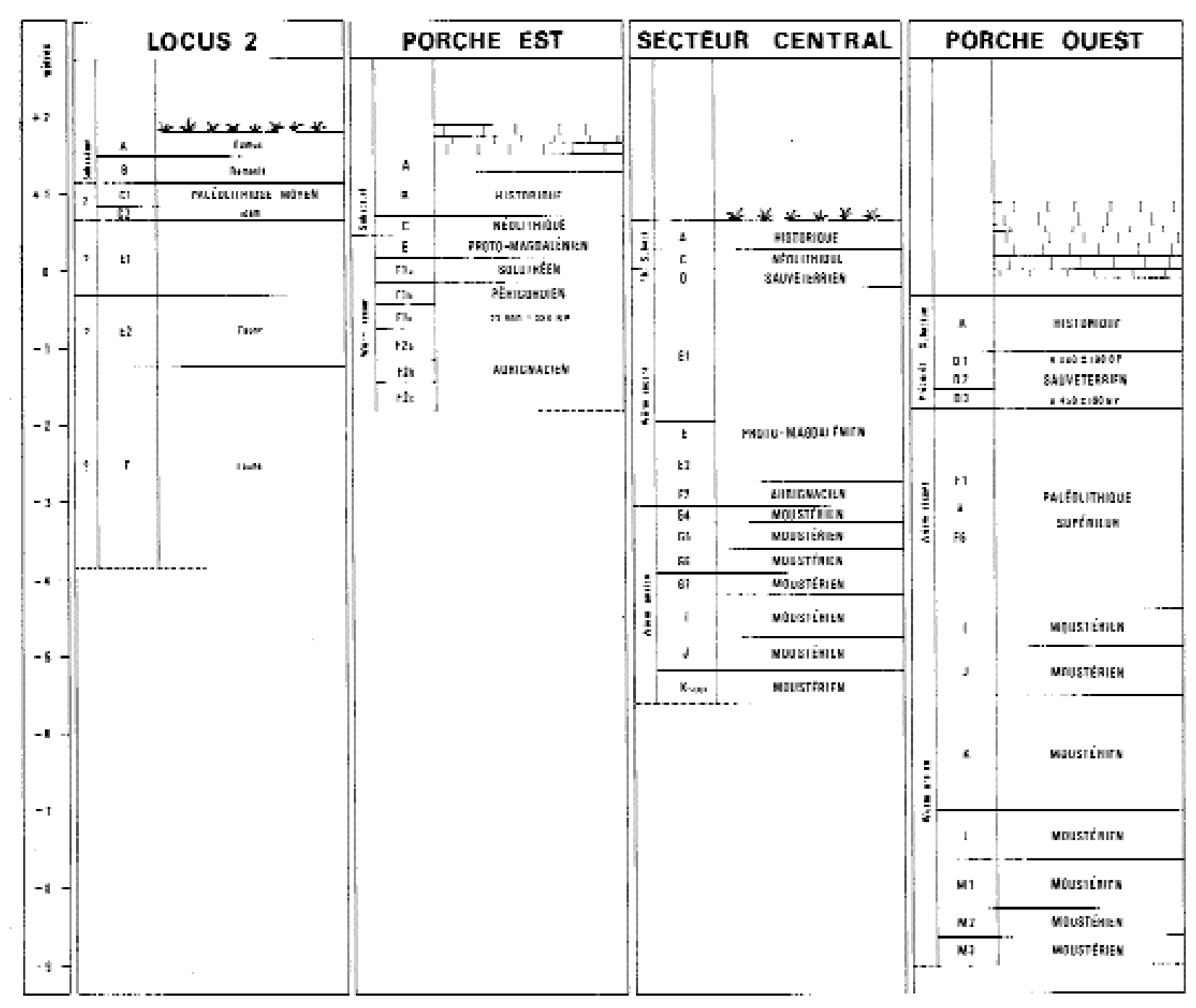

Figure 3 - Les différentes stratigraphies du gisement par secteur (d'après Champagne et al. 1990).

Figure 3 - Stratigraphy of the site described per area (Source: Champagne et al. 1990).

a été particulièrement utilisée pour étayer l'hypothèse de contemporanéité d'industries différentes, notamment l'Aurignacien et le Gravettien (Bordes 1968 ; Laville et Rigaud 1973 ; Rigaud 1976).

F. Champagne part du principe que le remplissage sédimentaire du gisement représente la totalité de la séquence chronologique. Les couches cryoclastiques correspondent alors au Paléolithique tandis que l'arrivée du limon traduit un changement climatique qui serait postérieur au Würm. Ainsi, l'industrie de la couche E serait épipaléolithique. C'est à partir de cette hypothèse que $\mathrm{J}$. Jaubert va mener ses comparaisons.

Cependant, depuis le début des fouilles de la couche E, F. Champagne mentionne le caractère périgordien de l'industrie, tout en insistant sur son originalité (Champagne 1976). Il propose alors deux hypothèses : la première est celle d'un nouveau faciès de transition Paléolithique-Mésolithique, en accord avec l'interprétation chronologique du remplissage. La seconde est celle d'un faciès local de tradition périgordienne, avec la restriction que " [...] dans cette hypothèse, il faudrait reconsidérer la datation de la castine sous-jacente " (Champagne 1976).

II y a donc, dès le début, une discordance entre l'interprétation chrono-climatique du remplissage et une industrie présentant manifestement des caractères gravettiens. J. Jaubert (1979), dans son étude de la couche E, reste attaché à cette interprétation du remplissage mais ne conclut pas sur l'attribution chrono-culturelle le problème demeure entier: "Nous sommes persuadés que la couche $E$ des Fieux ne peut être attribuée qu'à un faciès épipaléolithique local, étant entendu que nous utilisons le te rme Epipaléolithique pour situer dans le temps, une 

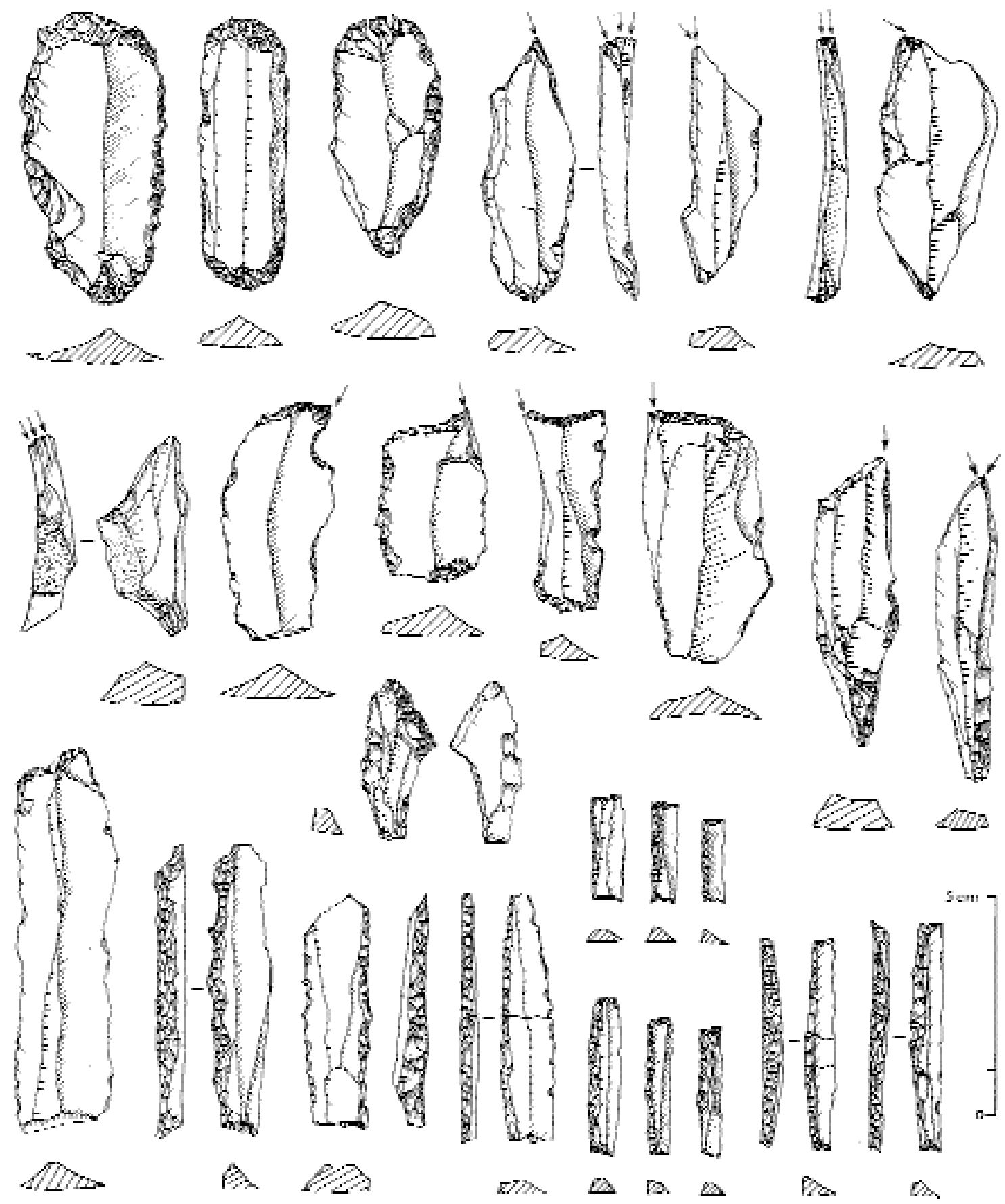

Figure 4 - Industrie lithique de la couche E présentée comme appartenant au Périgordien évolué ou Proto-Magdalénien (dessin N. Champagne, d'après Champagne et al. 1990).

Figure 4 - Lithical industry from the E layer and presented as belonging to the evolved Perigordien, or Proto-Magdalenien (Drawing: N. Champagne, Source: Champagne et al. 1990). 
industrie que tout incline à considérer comme contemporaine de l'extrême fin du Würm, mais qui n'a rien de commun avec le Mésolithique traditionnel " (Champagne et Jaubert 1979, p.98).

Ainsi, sur les bases d'une interprétation chrono-climatique du remplissage sédimentaire, F. Champagne s'attache à qualifier l'ensemble de la couche $\mathrm{E}$ d'épipaléolithique alors que son caractère gravettien avait été observé dès le départ.

1.2.3 - Une nouvelle hypothèse : l'attribution au Protomagdalénien.

Lorsqu'il présente la couche $\mathrm{E}$ au colloque de Bordeaux en 1977 sur la Fin des Temps glaciaires en Europe, F. Champagne est interpellé par D. de Sonneville-Bordes qui attribue l'industrie de la couche $\mathrm{E}$ au Périgordien. À la suite de cette discussion, F. Champagne décide de ne pas publier sa présentation (J. Jaubert, communication orale).

Presque dix ans plus tard, F. Champagne propose une nouvelle hypothèse d'attribution, évoquant un rapprochement avec le Protomagdalénien (Champagne 1986-1987). Le préhistorien rapproche alors l'ensemble de la couche $E$ de la famille gravettienne (fig. 4), tout en lui conférant un caractère récent, appuyé par l'existence d'un niveau solutréen sous-jacent (la couche F1a du porche est).

Les interprétations livrées par l'étude sédimentologique menée par N. Belounis semblent conforter cette hypothèse : le dépôt du limon se placerait immédiatement après l'épisode de Tursac. Une manifestation d'illuviation a par ailleurs été observée à mi-hauteur, attribuée à l'épisode de Laugerie (Belounis 1987). Cette demière détermination semble se confirmer dans les données de la palynologie (Renault-Miskovsky 1983), l'ensemble de ces résultats se basant sur les méthodes et les cadres chronologiques en vigueur à cette date.

Néanmoins, F. Champagne ne cache pas ses difficultés à conclure. II reprend les problèmes liés à une fourchette stratigraphique trop large, l'absence de vestiges osseux ou de restes calcinés empêchant la réalisation d'une datation radiométrique. Concernant l'étude des vestiges, il ajoute : " L'étude exhaustive du matériel lithique n'est pas encore terminée ; dans ces conditions il est prématuré de tenter une analyse comparative de la couche $E$ avec des séries datées du Périgordien final ou évolué et du ProtoMagdalénien. Il semble néanmoins que c'est vers cette dernière attribution culturelle que les comparaisons devront s'orienter [...]. En conclusion, l'ensemble lithique recueilli dans la couche $E$ est incontestablement de tradition périgordienne, mais il s'écarte franchement des faciès classiques de cette culture par l'absence de vraie pointe de la Gravette, de pointe de la Font-Robert et de burin de Noailles. Un essai d'analyse factorielle des correspondances tenté par $B$. Bosselin a montré que le niveau $E$ possède à la fois les caractères du Magdalénien et du Périgorden, mais les données de la sédimentologie et de la palynologie, et surtout sa position stratigraphique pro- bable au-dessus du Solutréen, en font une industrie nettement plus récente que le Périgordien évolué " (Champagne et al. 1990, p.16).

\section{3 - Reprise des données : la couche $\mathrm{E}$, un niveau gra- vettien au sein d'un gisement stratifié}

Conformément au souhait de F. Champagne, l'étude de l'ensemble de la couche $E$ est reprise dans le cadre d'un mémoire de DEA (Guillermin 2004), intégré dans l'ACR sur le Quercy. La série lithique bénéficie ainsi d'un nouveau regard porté sur le matériel par le biais d'une analyse typo-technologique. Cette reconsidération implique une reprise des données stratigraphiques des différents secteurs, afin de préciser le positionnement de la couche $\mathrm{E}$.

\subsection{1- Les caractères gravettiens de la couche $E$}

Plusieurs éléments permettent d'attribuer résolument toute une partie de la couche E au Gravettien. Certains peuvent être qualifiés de " typiquement " gravettiens, étant présents dans la quasi-totalité des assemblages attribués à cette tradition (fig. 5), à l'image de pointes et micropointes de la Gravette. Nous pouvons les associer à une modalité opératoire " classique " d'obtention de leur support, souvent qualifiée de "bipolaire ", qui sera décrite ultérieurement.

D'autres éléments sont plus spécifiques du faciès à burins du Raysse (fig. 6), ces derniers étant présents en quantité non négligeable. D'après les travaux récents (Klaric 2003 ; Klaric et al. 2002), il est par ailleurs possible de les associer à d'autres éléments caractéristiques, plus discrets au sein de l'ensemble : une lamelle de la Picardie, des lames portant une préparation particulière (dite à " facettage latéralisé oblique ") ainsi que des nucléus laminaires exploités suivant une modalité proche de la méthode décrite par L. Klaric.

Le niveau $\mathrm{E}$ des Fieux appartient au monde gravettien et peut-être plus particulièrement, sous réserve de son homogénéité, au Gravettien moyen. II est cependant nécessaire d'approfondir l'étude avant d'effectuer des comparaisons plus précises. En premier lieu, la question est de savoir quelle est sa relation avec les autres niveaux du gisement attribués à cette culture.

1.3.2 - Une réévaluation nécessaire de la stratigraphie du porche Est

La stratigraphie relevée dans le secteur Est montre la superposition de la couche $\mathrm{E}$ à un niveau solutréen (couche F1a), deux niveaux gravettiens (F1b et F1c) et un niveau aurignacien (couche F2) (fig. 7). La reprise des carnets de fouilles ainsi que des séries nous invite à une certaine réserve concernant l'intégrité de ces différents assemblages.

1.3.2.1- Des problèmes de distinction entre les différents niveaux

Les vestiges attribuables à la couche $E$ sont très peu nombreux dans le secteur Est où nous sommes à la limite de 

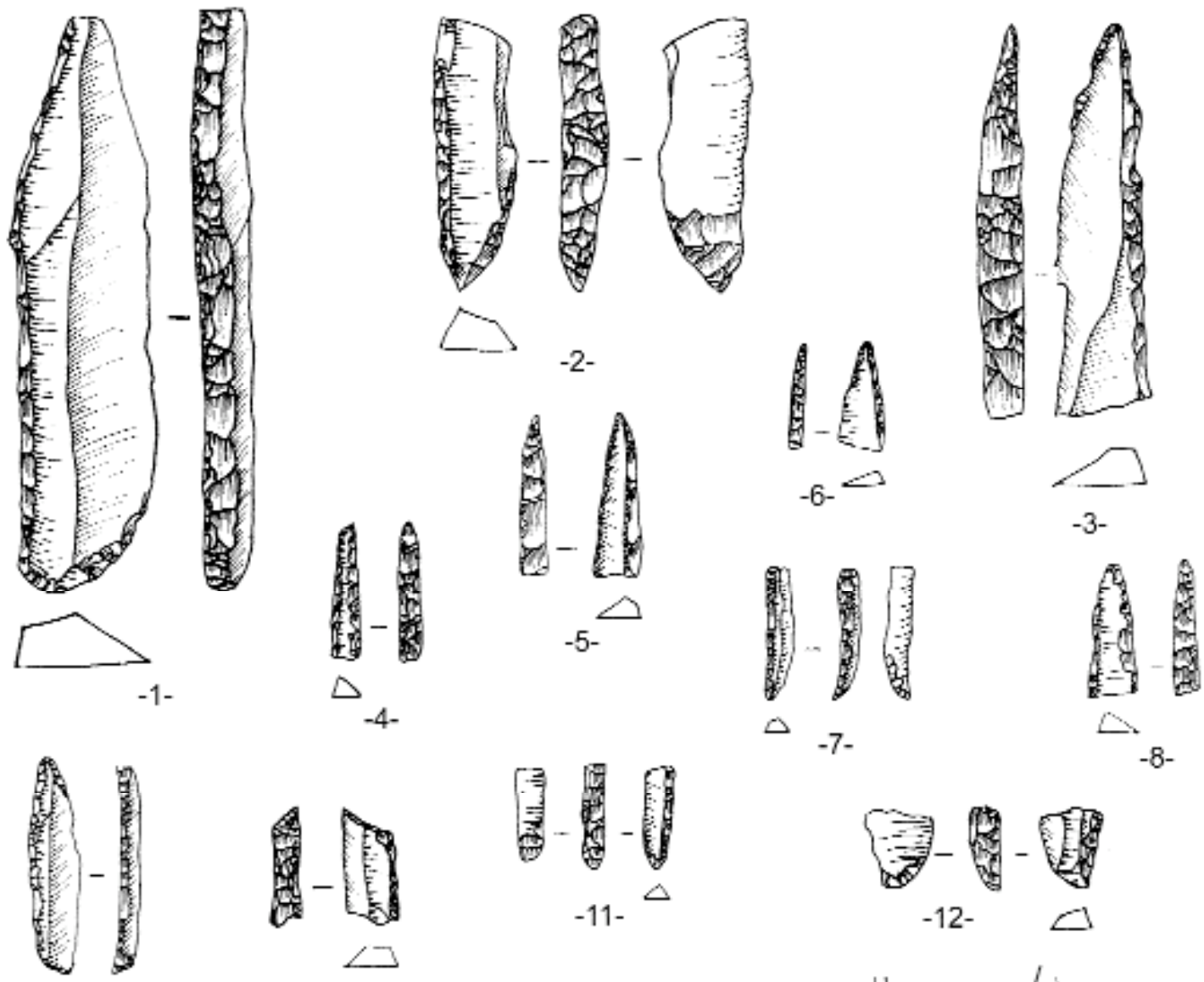

$-7-$
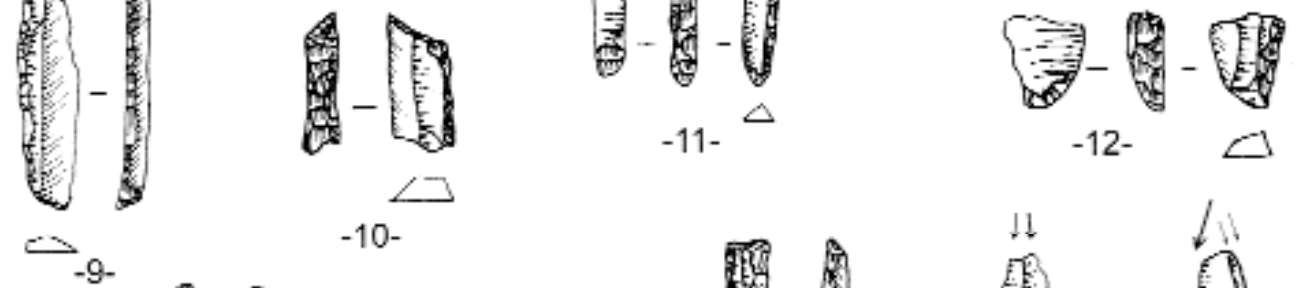

$-10-$
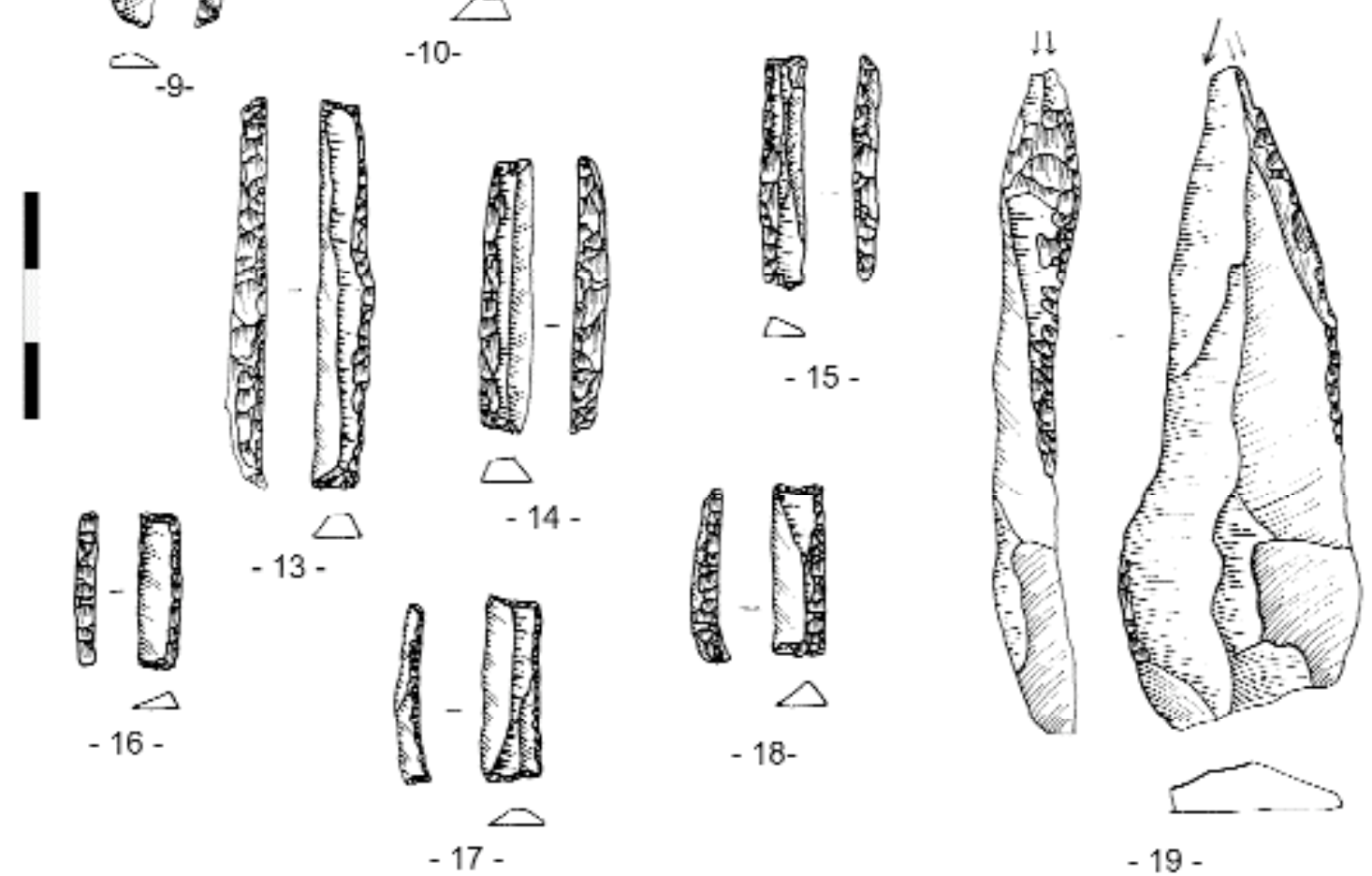

$-19-$

Figure 5 - Les amatures de la couche $E$ : pointes de la Gravette (1 à 3), microgravettes (4 à 12), lamelles à dos tronquées (13 à 18), lame appointée portant des fractures burinantes latérales (19).

Figure 5 - The projectile points of the E layer : Gravette's points (1 to 3), microgravettes (4 to 12), backed truncated bladelets (13 to 18), pointed blade showing traces of lateral hammering (19). 

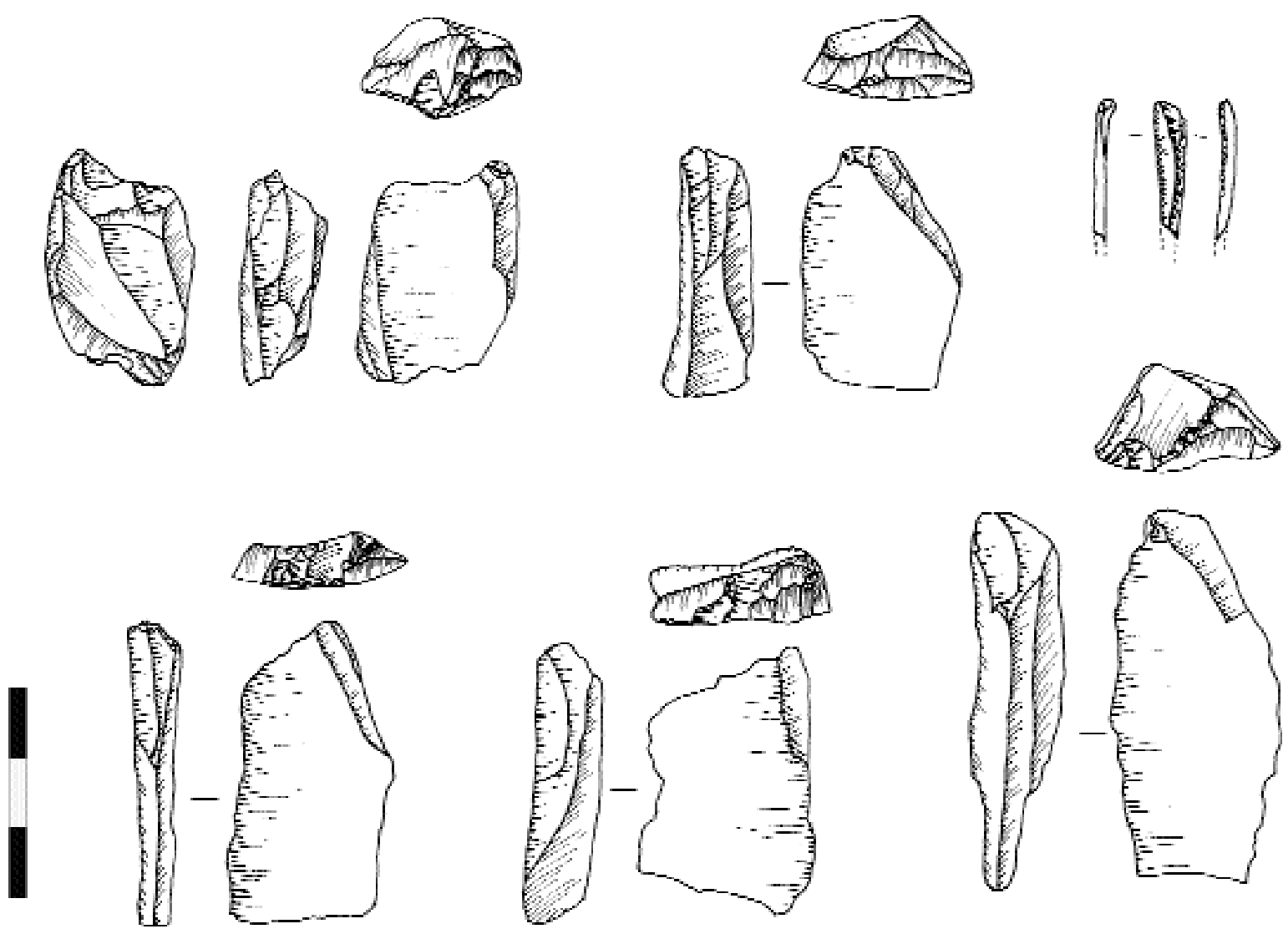

Figure 6 - Quelques éléments "rayssiens " de la couche E : une lamelle de la Picardie et des burins du Raysse.

Figure 6 - Some "rayssiens " elements from the E layer: one Picardie's bladelet and several "burins du Raysse”.
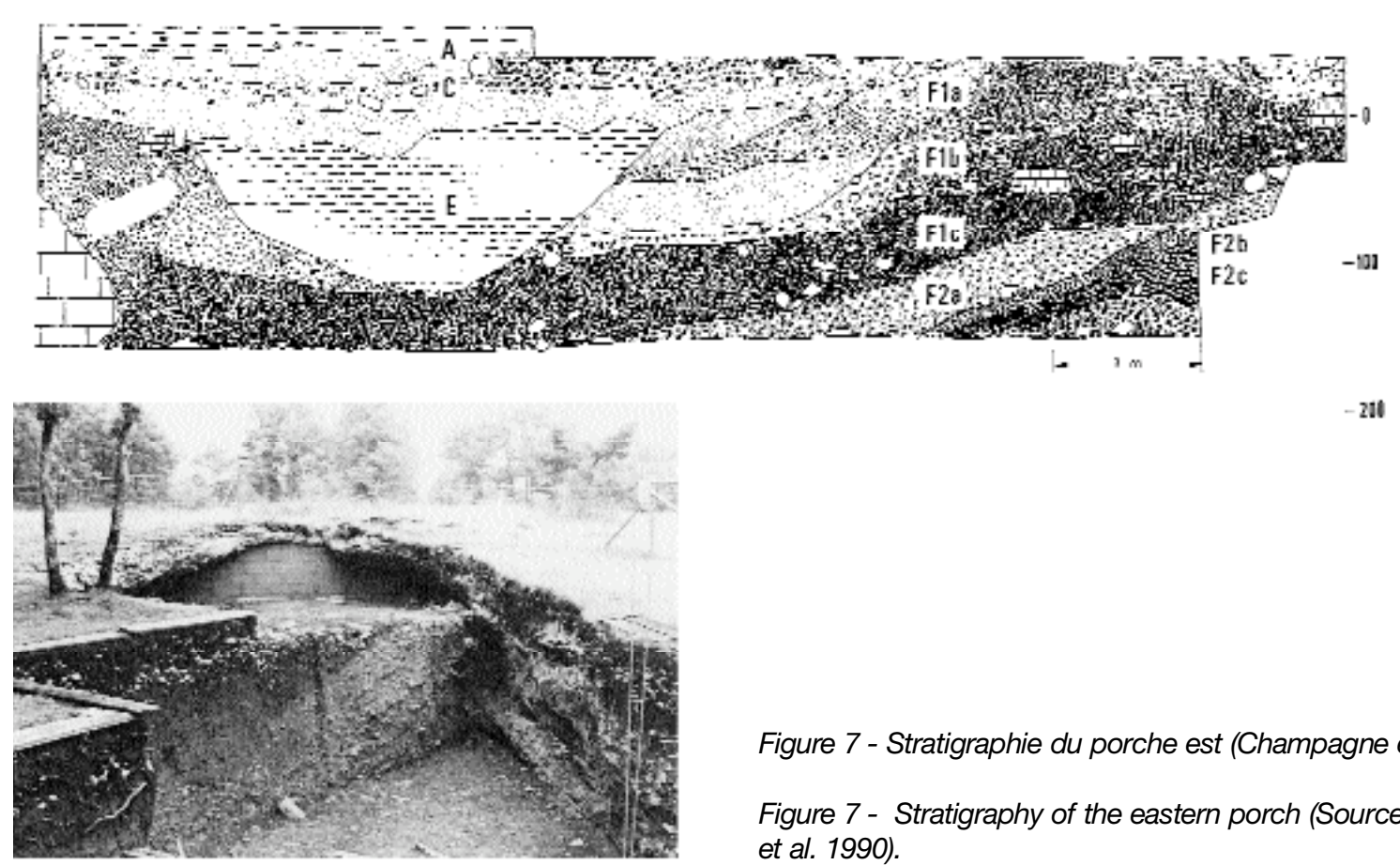

Figure 7 - Stratigraphie du porche est (Champagne et al. 1990).

Figure 7 - Stratigraphy of the eastern porch (Source: Champagne et al. 1990). 
l'extension de la couche. De plus, aux abords des parois, le niveau disparaît. À cet endroit, "le cailloutis cryoclastique remonte au niveau de la couche $C$ " (Champagne 1982). Lors de la découverte de la couche sous-jacente, F. Champagne écrit : " [...] immédiatement sous le dépôt de limon, elle est recouverte près des parois par un cailloutis composé d'éléments fins très altérés, probablement colluviés à partir de la surface du lapiaz. Nous avons décomposé arbitrairement $F 1$ en trois sous-niveaux :

-F1a : limite limon-castine

- F1b : partie supérieure de la castine

- F1c : niveau mieux individualisé à l'intérieur de la castine " D'après les 62 outils trouvés à ce stade des fouilles, F. Champagne avance une appartenance de la série au Périgordien (Champagne 1983).

La stratigraphie du porche Est montre la succession de couches proches de la paroi, dans des sédiments cryoclastiques qualifiés d'éboulis, à très fort pendage. Les subdivisions au sein de ces sédiments, faites de manière arbitraire au départ, ont connu par la suite une interprétation archéologique. Les notes des carnets de fouilles montrent que les fouilleurs ont eu du mal à les discemer. De plus, ces distinctions ont été conservées lors de l'extension de la fouille vers le porche Est.
1.3.2.2 - Remise en cause de la superposition de la couche $\mathrm{E}$ au niveau solutréen $\mathrm{F} 1 \mathrm{a}$

F. Champagne attribue la couche F1a au Solutréen par la présence de deux pointes à face plane, de deux lames appointées, d'un grattoir circulaire et d'un fragment de sagaie (fig. 8).

Cette couche est constituée d'un dépôt limono-argilosableux associé à des éléments calcaires de petites dimensions. Le reste de l'outillage est composé de burins, lames retouchées et pièces à dos qui ont, pour la plupart, un caractère gravettien. En outre, les cinq éléments solutréens cités ont été trouvés lors de l'extension des fouilles sous le porche, à un endroit où la couche $E$ n'est plus présente (fig. 8). Ainsi, les témoins d'une occupation solutréenne aux Fieux sont faibles et mélangés à des éléments gravettiens. En définitive, à l'endroit où la couche $\mathrm{E}$ se superpose au niveau F1a, celle-ci ne contient pas d'éléments caractéristiques du Solutréen.

1.3.2.3 - Les couches F1b et F1c : des niveaux gravettiens stratifiés

Le sédiment de la couche F1b est de teinte brun-jaune et contient de petites plaquettes calcaires associées à des
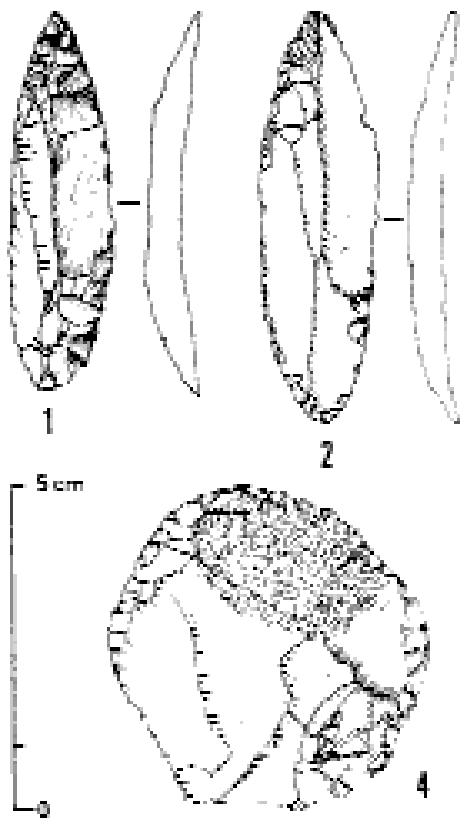

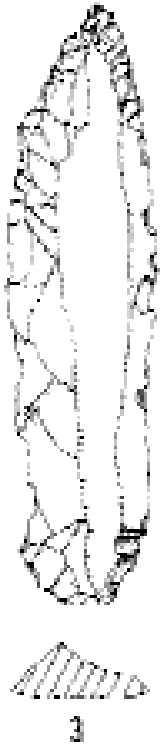

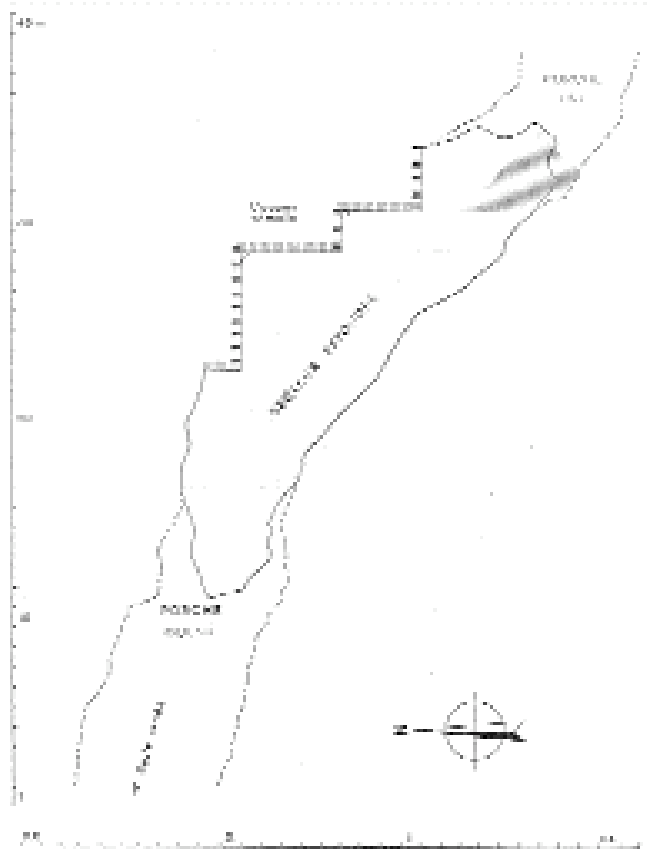

Figure 8 - A gauche : les outils solutréens de la couche F1a; à droite : la zone hâchurée correspond à l'extension des fouilles pour dégager le porche est, les outils solutréens ont été trouvés à cet endroit alors que la couche $E$ n'est plus présente.

Figure 8 - Left: solutrean tools from the F1a layer; Right: the hatched area corresponds to the excavation extension to clear the eastern porch. The solutrean tools were found in this area where the $E$ layer is not in place anymore. 


\begin{tabular}{|c|c|c|c|}
\hline Liste typologique & F1a & F1b & F1c \\
\hline grattoir & 3 & 3 & 5 \\
\hline burin & 15 & 14 & 16 \\
\hline burin du Raysse & & 1 & 3 \\
\hline pointes de la Gravetle & & 2 & 1 \\
\hline microgravetto & 1 & 7 & \\
\hline lame à dos & 3 & & 4 \\
\hline lame retouchee & 6 & & 1 \\
\hline piéce esquillèe & 1 & 3 & \\
\hline lamelle à dos & 4 & 10 & 9 \\
\hline lamelle à dos tronquéce & & 3 & 2 \\
\hline lamollo à dos bitronquéc & 1 & 1 & \\
\hline lamelle tronquée & 1 & & \\
\hline lame apointèe & 2 & & \\
\hline poinle á lace plane & 2 & & \\
\hline divers & & 4 & 5 \\
\hline total & 39 & 48 & 46 \\
\hline
\end{tabular}

Tableau 1- Inventaire typologique des industries des couches F1a, F1b et F1c du porche Est.

Table 1 - Typological inventory of tools from layers F1a, F1b and F1c coming from eastern porch.
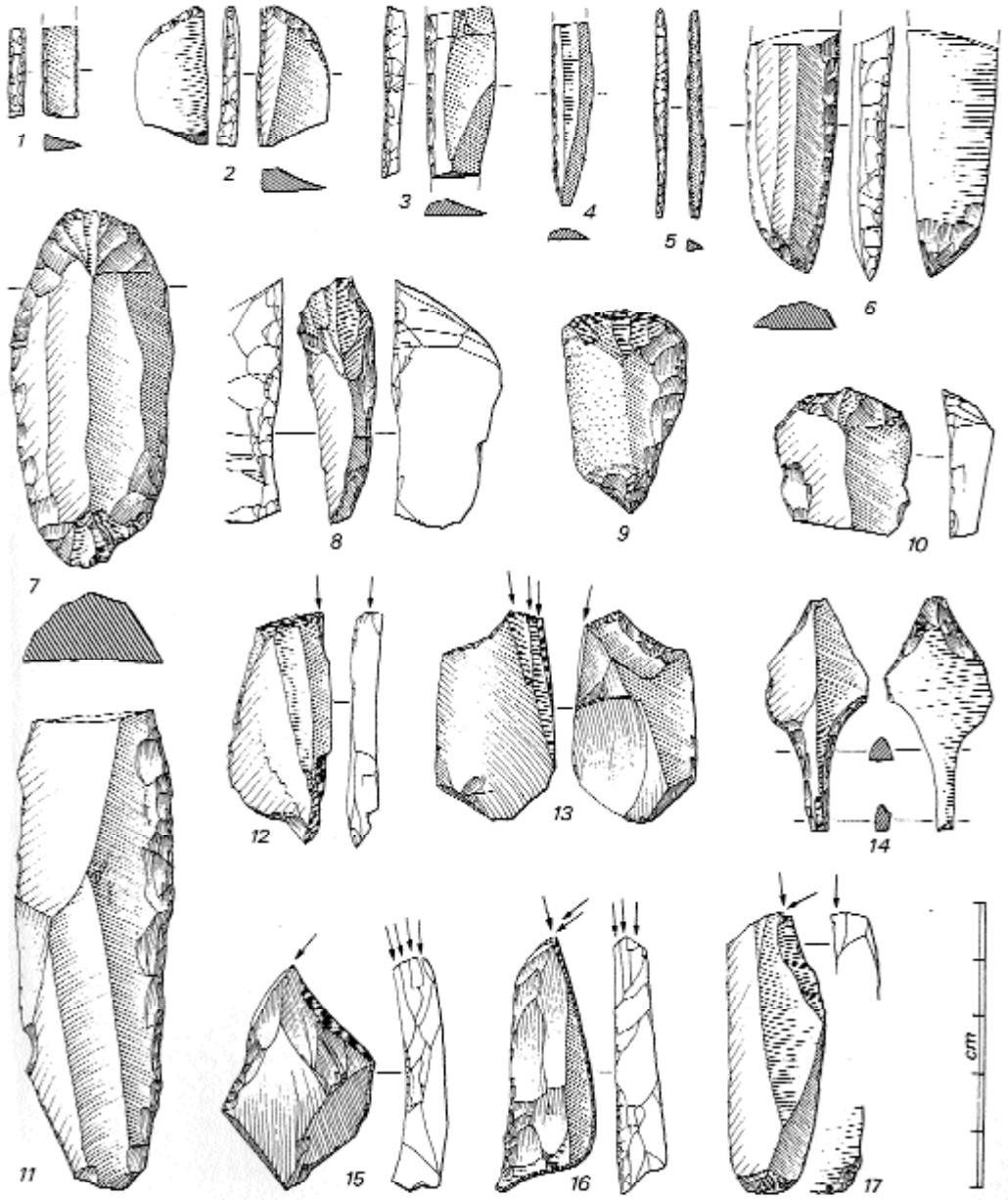

Figure 9 - Industrie de la couche $F$ (d'après Champagne et al. 1979).

Figure 9 - Industry from the $F$ layer (Source: Champagne et al.1979). 
cailloux de forme polyédrique. La série lithique recueillie dans ce niveau est pauvre mais la présence de pointes de la Gravette permet de l'attribuer au "Périgorden supérieur à pointes de la Gravette " (Champagne et al. 1990).

Concemant la couche F1c, F. Champagne écrit : "De composition sédimentologique et de teinte identiques, les couches $\mathrm{F} 1 \mathrm{~b}$ et $\mathrm{F} 1 \mathrm{C}$ sont difficiles à isoler, sauf vers la para sud où elles sont séparées par des blocs issus de l'effondrement de la voûte [...] Le matériel lithique comp rend 69 outils et 536 lames et éclats. Nous attribuons cette série à un faciès du Périgordien moyen, encore difficile à préciser et qui semble dépourvu de pointe de la
Gravette typique et de burin de Noailles. Cette attribution est par ailleurs confirmée par une mesure d'âge qui a donné $23900 \pm 330$ BP " (Champagne et al. 1990, p.17). II ajoute que la faune est assez riche, comprenant en grande partie des fragments de bois de renne en très mauvais état de conservation "dû à l'infiltration de la couche E" (Ibid.).

L'observation des séries révèle des industries à effectif restreint se démarquant très peu de l'outillage de la couche E, comme nous le verrons par la suite (tab. 1) : les armatures, variées, sont bien représentées et le burin du Raysse est présent.

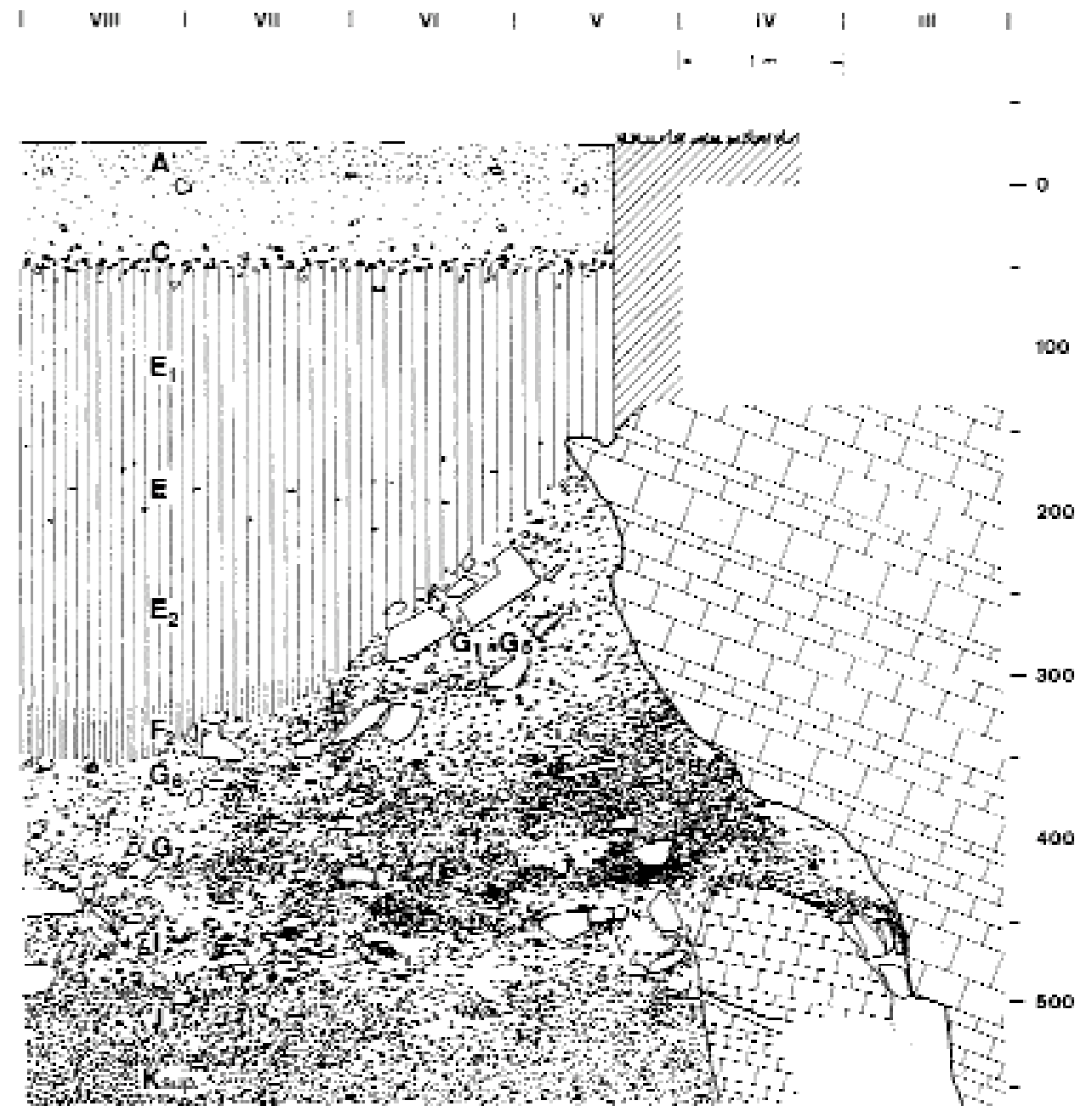

Figure 10 - Stratigraphie du secteur central, coupe frontale 21-22 : A, humus ; C, Néolithique ; E, niveau gravettien, E1 et E2 représentent les ensembles d'artéfacts “flottants " au-dessus et en-dessous du niveau; F2, Aurignacien; G1 à K, Moustérien.

Figure 10 - Stratigraphy of the central area, front cross section 21-22 : A, humus ; C, Neolithic ; E, gravettian layer, E1 and E2 represent the groups of "floating" artefacts above and bellow the layer, F2, Aurignacian; G1 à K, Mousterian. 
Au final, une étude taphonomique et typo-technologique plus approfondie des assemblages lithiques doit être menée avant d'utiliser les données stratigraphiques de ce secteur.

\subsection{3 - Une contamination de l'Aurignacien dans le secteur} central

La quasi-totalité des vestiges de la couche $\mathrm{E}$ sont concentrés dans le secteur central, se superposant à un niveau aurignacien dans la moitié est du secteur (fig. 10). Or, quelques éléments à caractère aurignacien, identiques à l'industrie de l'Aurignacien récent du niveau F2 du secteur Est (Bon et Bordes 2005) (burins busqués, grattoir à museau, lame aurignacienne, lamelles Dufour) sont présents dans l'assemblage de la couche $\mathrm{E}$.

Nous avons écarté ces éléments de l'étude $(n=18)$. D'autres, plus ubiquistes, tels que des burins carénés - pouvant être considérés comme des burins busqués "atypiques "- ont également été mis de côté, sans pouvoir être attribués de manière certaine à l'une ou l'autre des industries $(n=13)$.

1.3.4 - Une pointe de la Font-Robert trouvée dans le secteur ouest

Beaucoup d'incertitudes demeurent dans ce secteur. Les différentes industries du Paléolithique supérieur trouvées au sein de l'important ensemble de castine $(F)$, " n'ont pu être isolées avec certitude " (Champagne 1974). En effet, les couches de ce secteur sont pour la plupart composées de plaquettes calcaires emballées dans un sédiment arg ilo-limono-sableux dont la teinte varie du brun-rouge au brun foncé et il a été difficile de différencier différentes unités à l'intérieur.
Ainsi, tous les vestiges trouvés ont été regroupés dans un ensemble intitulé couche $F$, qui a livré un marqueur fort du Gravettien : une pointe de la Font-Robert (fig. 9). En réalité, la série révèle une association d'éléments aurignaciens (essentiellement des burins busqués, lames aurignaciennes) et gravettiens (pièces à dos, burins du Raysse et la pointe de la Font-Robert). Les premiers sont présents de manière ponctuelle sur toute la surface du secteur, tandis que les seconds sont plus concentrés, proches du massif stalagmitique.

En ce qui concerne la couche $E$, son étendue ainsi que sa superposition à la couche $F$, ne sont pas clairement établies. II est possible que la distinction sédimentaire entre les deux couches ne soit pas apparue de manière toujours évidente, le limon pouvant être associé, par endroit, à des éléments calcaires.

En conclusion, la reprise des données stratigraphiques des différents secteurs du gisement des Fieux appelle à une certaine réserve concernant les interprétations de $\mathrm{F}$. Champagne : d'une part, il n'est plus possible d'entériner la superposition de la couche $E$ à un niveau solutréen ; d'autre part, les difficultés de lecture stratigraphique de niveaux gravettiens comportant de faibles indices, nous laissent, pour l'instant, peu de données exploitables.

\section{4 - Les questions taphonomiques relatives à l'étude de la couche $E$}

La présence de mobilier aurignacien dans la couche $\mathrm{E}$, ainsi que les méthodes de fouilles de l'époque ne facilitant pas la lecture du pendage des couches, sont autant
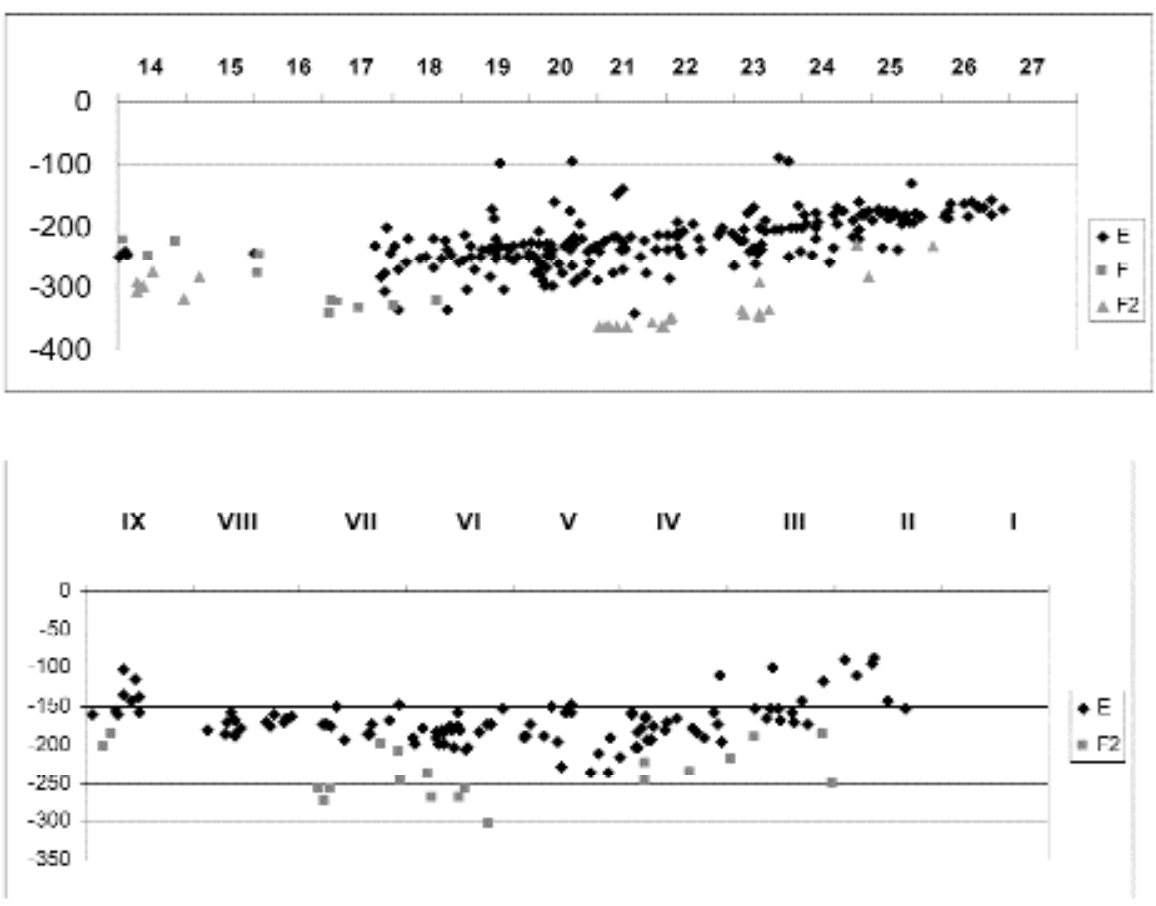

Figure 11 - Projection verticale des outils côtés à la fouille. En haut : bande VIII, en dessous : bande 26, en bas à droite : raccords de débitage effectués sur le matériel.

Figure 11 - Vertical projection of the tools measured during the excavation. Above : stripe VIII, bellow : stripe 26, Bottom right: refittings. 
d'éléments conduisant à s'interroger sur l'intégrité de la série par rapport aux autres niveaux archéologiques. Par ailleurs, la répartition du matériel au sein d'un puissant dépôt sédimentaire d'une épaisseur exceptionnelle de plus de trois mètres, demande à être précisée.

\subsection{1- Une concentration de vestiges dans le secteur central}

Étant donnés les problèmes énoncés précédemment dans les secteurs est et ouest, nous avons choisi de restreindre le corpus d'étude au secteur central (des bandes 17 à 26, fig. 11). Cette restriction n'a qu'une incidence minime sur l'assemblage (près de $10 \%$ ), la majorité des vestiges étant concentrée dans ce secteur.

La densité de mobilier est par ailleurs visible dans l'épaisseur du sédiment, révélant l'existence d'un niveau archéologique (fig. 11). Cette concentration était telle que $F$. Champagne, parlant de la découverte d'un " sol d'habitat ", change ses méthodes de fouilles et entreprend un grand décapage du niveau à l'occasion du Congrès Préhistorique de France en 1979 (Champagne et Jaubert 1981).

\subsection{2- Raccords et remontages}

Des raccords de cassure et de débitage ont été effectués. Ils concernent essentiellement les silex jaspéroïdes, l'importance de la série et les contraintes de temps ne nous ayant pas permis d'intégrer l'ensemble des vestiges dans cette démarche. Au total, 1350 pièces ont été considérées, le taux de raccord de débitage (calculé ici à partir du nombre d'artefacts raccordés sur le nombre total d'artefacts) s'élève à $11 \%$. L'espace couvert par ces raccords révèle une très nette concentration correspondant au " sol d'habitat" (fig. 11).

Ceci confirme l'existence d'un niveau archéologique (mais pas nécessairement d'un sol d'habitat) dans le secteur central. Son épaisseur est d'environ $30 \mathrm{~cm}$, quelques vestiges isolés 'flottant' au-dessus et en dessous (à partir de 1981, les vestiges trouvés sous le " sol d'habitat " sont notés E2) (fig. 11). Ce niveau accuse un pendage fort, marqué d'est en ouest, et épouse transversalement la forme d'un " paléochenal ", le matériel remontant aux abords des parois.

Nous ne pouvons conclure sur le degré de perturbation de ce dépôt archéologique. Nous reprenons néanmoins l'affirmation posée par D. Cahen et al. : " La présence de pièces raccordables à un même niveau indique généralement que la couche n'a pas été sérieusement perturbée " (Cahen et al. 1980, p.212).

Nous posons ainsi l'hypothèse de l'intégrité de notre corpus d'étude par rapport aux autres occupations gravettiennes distinguées par les fouilleurs. L'étude du matériel nous permettra par la suite d'avancer des arguments en faveur de son homogénéité.

Tableau 2 - Inventaire typologique d'après la liste type de D. de Sonneville-Bordes et J. Perrot (Sonneville-Bordes et Perrot 1954, 1955 et 1956).

Table 2 - Typological inventory according to type-list written by $D$. de SonnevilleBordes et J. Perrot (Sonneville-Bordes et Perrot 1954, 1955 et 1956).

\begin{tabular}{|c|c|}
\hline $\begin{array}{l}\text { catégorie typologique } \\
\text { gratloir sur lame }\end{array}$ & $\begin{array}{r}\text { cffectif } \\
10\end{array}$ \\
\hline grattor abyolque & 14 \\
\hline grattoir doukhis & 2 \\
\hline grattibir cgival & 1 \\
\hline grattoir sur lame refouchèe & 18 \\
\hline grattoir sur éclat & 10 \\
\hline grattoir carene atyolque & 5 \\
\hline grattnir-burin & 5 \\
\hline percoir atypizus & 7 \\
\hline burin diedre droit & B \\
\hline burin diedre dejelte & 8 \\
\hline bunn diedre o'engle & 8 \\
\hline hurin sur rassure & 22 \\
\hline hurin dicdre multipls: & 7 \\
\hline burin sur troncature droite & 4 \\
\hline burin sur troncature oblicue & 10 \\
\hline bunn sur troncature concave & 25 \\
\hline hurin sur troncature conwexe. & 10 \\
\hline $\begin{array}{l}\text { bunn sur troncature } \\
\text { transversale }\end{array}$ & 1 \\
\hline burin mulliple mixte & 10 \\
\hline burin mulliple mixte & 12 \\
\hline bunn nuclaitorme & 2 \\
\hline hurin plan & 22 \\
\hline burin du Raysses & 23 \\
\hline pointe de la Gravetle & 17 \\
\hline pointe de la Gravetle stypique & 3 \\
\hline microgravette & 36 \\
\hline lame ì dos & 24 \\
\hline lame ì dos tranquês: & B \\
\hline lame as troncalure drote & 3 \\
\hline lame $a$ troncalure oblique & 11 \\
\hline lame a tronceture conceve & 8 \\
\hline lame ì troncature convexe & 1 \\
\hline lame bitronquiese & 2 \\
\hline lame \& retouche $\dot{a}$ un bord & 18 \\
\hline lame ds retouche a deux bords & 25 \\
\hline encoche & 25 \\
\hline denticulé & 4 \\
\hline piúcي esquilèt & 34 \\
\hline racloir & 1 \\
\hline lamelle tronquee & 6 \\
\hline Iamelle a dos & 151 \\
\hline lamsile ì ros tronquice & 102 \\
\hline lamelle is dus bitronguese & 25 \\
\hline lamulle is usu partiel & 4 \\
\hline lamelle retouches & 8 \\
\hline lame soontes & 17 \\
\hline divers: & 21 \\
\hline Total & 805 \\
\hline
\end{tabular}




\begin{tabular}{|l|r|}
\hline Outils & effectifs \\
\hline graltoirs/perçoirs & 65 \\
\hline burins & 114 \\
lamos rotouchees & 76 \\
\hline pièces esquillees & 34 \\
\hline èclats retouchès/racloirs/encoches/denticuless & 101 \\
\hline divers & 21 \\
\hline armatures & 384 \\
total & 805 \\
\hline
\end{tabular}

Tableau 3 - Outillage de la couche $E$ (les éléments considérés ne sont pas exactement les mêmes que ceux de l'inventaire typologique, le fait d'arriver au même total de l'effectif est un hasard).

Table 3- Tools from the $E$ layer (the artefacts considered are not identical to those mentionned in the typological inventory, similar results are pure coïncidence).

\section{2 - ETUDE TYPO-TECHNO-ECONOMIQUE DU MATÉRIEL LITHIQUE}

La série se compose de plusieurs milliers d'artefacts, F. Champagne parle de plus de 21000 objets, comptant les esquilles. Le corpus restreint au secteur central correspond donc à environ 19000 artefacts, dont plus de la moitié sont inférieurs à $1 \mathrm{~cm}$.

\section{1 - Un ensemble marqué par la présence d'amatures}

Deux catégories sont distinguées au sein de l'outillage : les amatures et les outils à usage domestique. L'inventaire typologique révèle l'importance de la première dont la production de supports constitue l'objectif principal du débitage (tab. 2 et 3 ).

\subsection{1- Diversité des armatures}

Les armatures de la couche E sont diversifiées (fig. 5) : certaines, minoritaires, sont sur support laminaire d'assez grand gabarit mais la plupart sont microlithiques, les supports pouvant être aussi bien de petites lames que des lamelles, voire exceptionnellement des chutes de burin. Les pointes et micropointes de la Gravette, déjà évoquées, sont bien représentées (fig. 12). Elles sont associées à des lamelles à dos tronquées, parfois bitronquées, presque aussi nombreuses que les lamelles à dos simples. Ces dernières présentent des modules variés parfois très petits. Signalons que l'absence de tamisage durant la fouille a vraisemblablement entraîné une sous-représenta tion de cette fraction la plus fine.

Par ailleurs, certains outils, initialement considérés comme des burins, peuvent être requalifiés de pointes : ce sont des lames apointées portant des fractures interprétables comme d'impact latérales burinantes.

2.1.2 - Beaucoup d'armatures et des fractures d'impact : des éléments appuyant l'hypothèse de spécialisation de l'occupation.

La proportion des armatures est importante au sein de l'outillage (près de $50 \%$ ). Les critères décrits et précisés sur les fractures d'impact des gravettes et microgravettes de Corbiac (O'Farrell 1996 et 2000), nous ont permis d'évaluer l'importance de ce type de stigmates (35\%). Par ailleurs, les extrémités apicales de pointes et micropointes de la Gravette de la couche E, portant pour certaines des fractures d'impact, ont pu être introduites par l'intermédiaire des carcasses ramenées sur le site, à l'intérieur desquelles elles étaient restées fichées (Chadelle et al. 1990). Ainsi, même si l'os n'est pas conservé, plusieurs éléments laissent envisager l'importance d'une activité de chasse conduite aux abords de l'occupation. Cela permet d'avancer l'hypothèse d'une interprétation de la couche $\mathrm{E}$ des Fieux comme résultant, au moins, d'une occupation spécialisée dans l'activité cynégétique.

\section{2 - Des schémas opératoires divers}

En dehors des nucléus informes ne révélant aucune modalité d'exploitation précise $(n=15)$, quatre principales formes de débitage lamino-lamellaire sur bloc ont été observées, nous les rapprocherons dans un second temps du débitage sur tranche d'éclat. Ces modalités sont par ailleurs accompagnées d'un débitage d'éclats plus ou moins allongés.

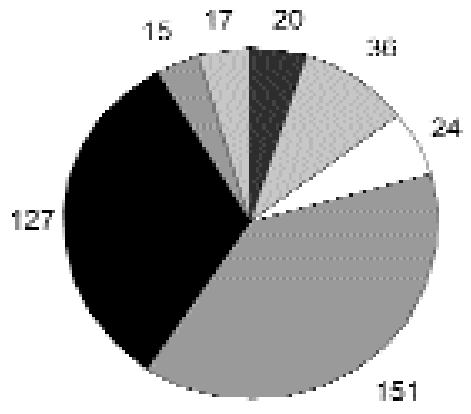

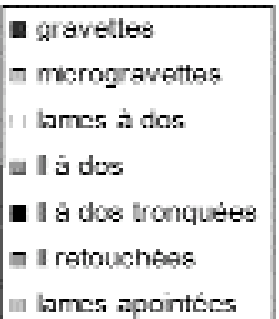

Figure 12 - Effectifs et proportions des différentes armatures de la couche $E$.

Figure 12 - Quantities and statistics of the various supports issued from the $E$ layer. 


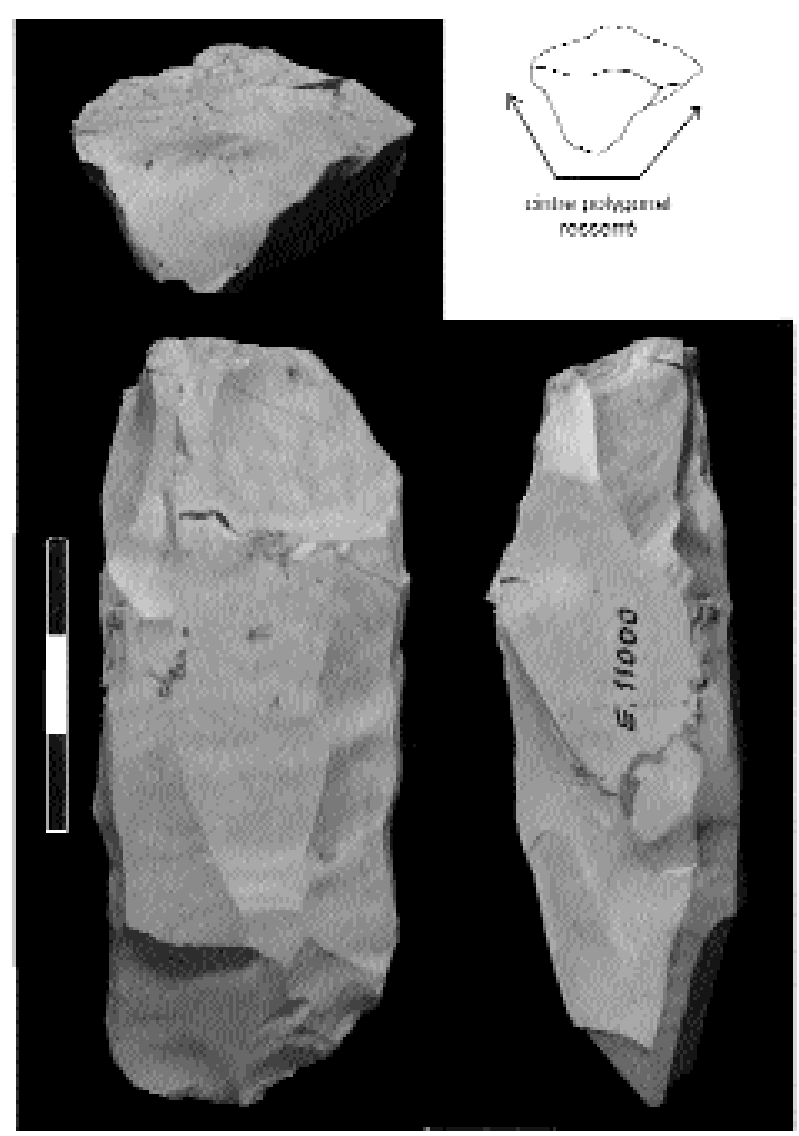

2.2.1 - Une exploitation frontale d'un volume au cintre polygonal resserré à l'aide de deux plans de frappe (fig. 13) $(n=16)$ :

C'est le schéma opératoire qui semble le plus communément rencontré dans un contexte gravettien, souvent qualifié de débitage bipolaire prismatique. Son objectif est la production de lames plus ou moins grandes mais toujours rectilignes et régulières, supports potentiels de pointes et micropointes de la Gravette.

L'initialisation du débitage peut se faire par l'extraction de lame à crête ou par envahissement progressif de table par des enlèvements plus ou moins obliques par rapport à l'allongement de la future surface de débitage. La mise en forme du volume au cintre polygonal est le plus souvent élaborée à l'aide de crêtes postéro-latérales. Certaines plages corticales peuvent par ailleurs être conservées tout au long de la séquence suivant le volume offert au départ par le bloc.

L'angle recherché entre les flancs et la table est relativement fermé, donnant un caractère resserré au cintre. Son entretien est réalisé à partir de crêtes mais aussi par l'enlèvement de grandes lames de flanc. Le rythme de recul frontal maintient des nervures saillantes donnant l'aspect polygonal au cintre.

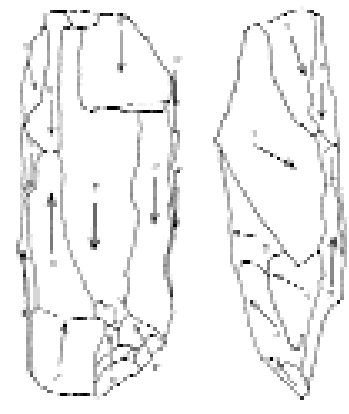

Figure 13 - Exploitation d'un volume au cintre polygonal resserré à l'aide deux plans de frappe.

Figure 13 - Exploitation of a volume with tightened polygonal "cintre" by the mean of a double napping platform.

Les plans de frappe sont majoritairement lisses L'installation du second semble intervenir dès le début du débitage. Cette double ouverture sur la table permet l'obtention de supports rectilignes. Très souvent, les plans de frappe sont décalés : l'exploitation à partir d'un premier plan de frappe entretient le cintre d'un côté de la table, favorisant l'exploitation de l'autre côté à partir du second. L'altemance de l'exploitation d'un bord à l'autre de la table, favorise pleinement l'entretien d'un cintre polygonal resserré.

Cette alternance des plans de frappe est plus visible sur les négatifs d'enlèvements des nucléus en fin d'exploitation que sur les supports d'outils ou sur les produits bruts. II semble donc que, dans la mesure où le débitage ne rencontre pas de difficultés, le tailleur ne recherche guère une utilisation simultanée des deux plans de frappe. En outre, les négatifs d'enlèvement opposés révèlent plutôt une hiérarchisation des plans de frappe qui peut s'inverser suivant les opportunités choisies par le tailleur durant la séquence. La question se pose alors de savoir à partir de quel rythme d'inversion il est possible de parler de débitage bipolaire.

Quoi qu'il en soit, l'exploitation d'un tel volume favorise avant tout la rectitude et la régularité des produits. De manière plus spécifique, elle privilégie l'épaisseur des supports tout en maîtrisant leur largeur, accentuant leur robustesse. 

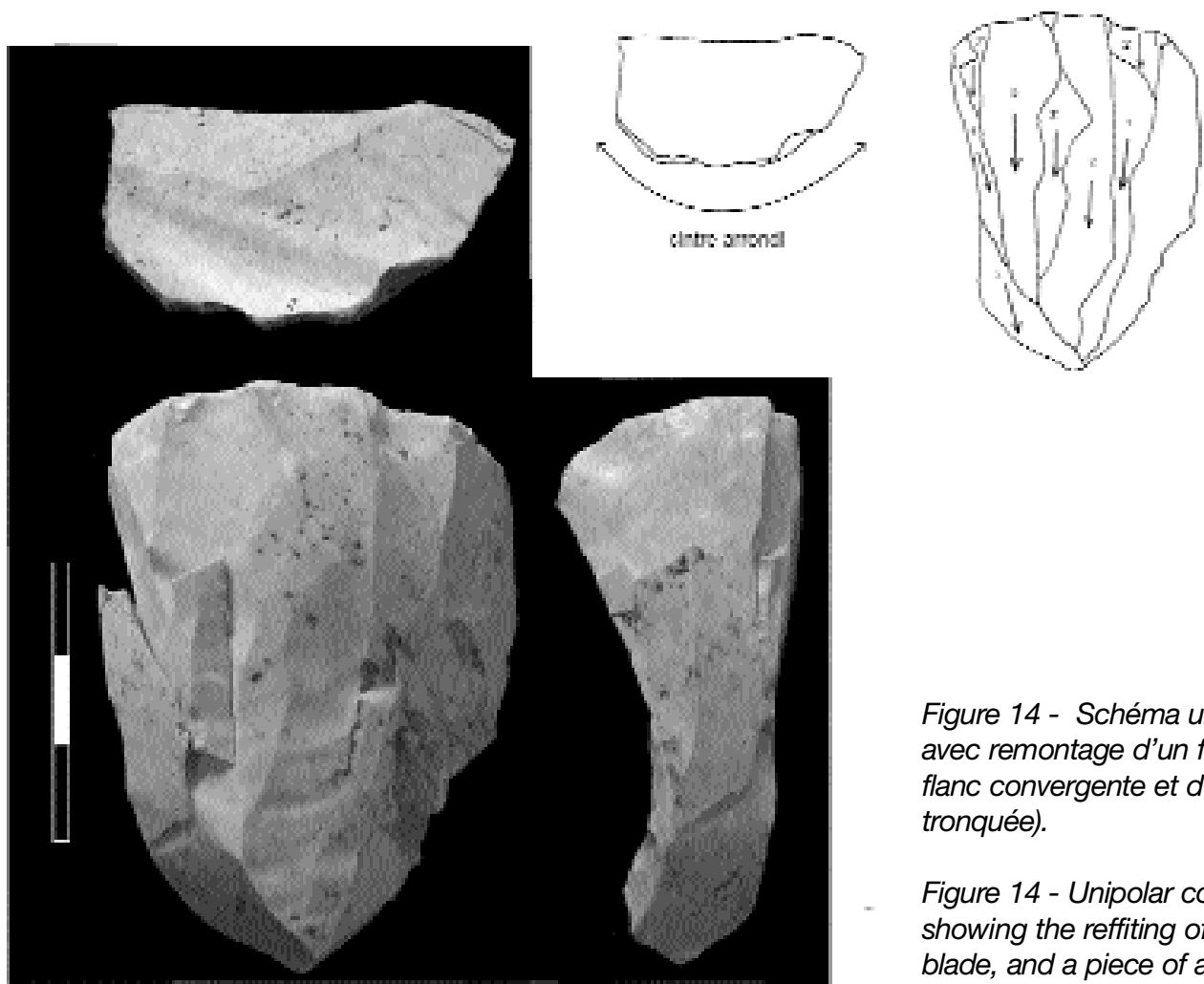

Figure 14 - Schéma unipolaire convergent (photo avec remontage d'un fragment distal de lame de flanc convergente et d'un fragment de lamelle à dos tronquée).

Figure 14 - Unipolar converging schema (picture showing the reffiting of a part of a side converging blade, and a piece of a backed truncated bladelet.

\subsection{2 - Une modalité unipolaire convergente (fig. 14) $(n=14)$}

L'objectif de cette modalité est également la production de supports laminaires (le plus souvent de petit gabarit) recti lignes et réguliers mais qui semblent toutefois moins épais que les produits issus du schéma précédent. Celui-ci repose en grande partie sur l'aménagement du cintre, dans le cadre d'une production gérée à l'aide d'un seul plan de frappe (le plus souvent lisse).

En effet, dans le cadre d'un débitage de produits recti lignes, la carène est plate sur une bonne partie de la table. Ainsi, le maintien du cintre est d'autant plus important : il permet de 'cadrer' la propagation de l'onde de choc, lui évitant de s'étaler. II est ici réalisé à l'aide d'enlèvements obliques convergents vers la partie distale de la table, issus de l'arrière du plan de frappe. De larges enlèvements transversaux issus de la table et/ou du dos peuvent s'associer à ces enlèvements obliques. L'angle alors formé entre les flancs et la table est relativement fermé.

Des lames sont par la suite débitées à cette jonction, recréant des nervures régulières pour guider les futurs enlèvements. Ainsi, à l'entretien du cintre, s'associe une entreprise de "nervuration " de la table, contrôlant l'étalement de l'onde de choc. Ces lames latérales sont souvent torses car débitées en retrait du plan de frappe au niveau du flanc et recentrées sur la table au niveau de leur partie distale. Sans être systématiquement torses, leur talon est très souvent incliné par rapport à l'axe de la pièce.

Cette opération a pour effet d'installer des nervures régulières, moins inclinées que celles installées par les premiers enlèvements plus obliques. L'installation de nervures- guides, fréquemment convergentes, prime sur le maintien d'une angulation fermée entre la table et les flancs. Le cintre recherché est ainsi arrondi en partie proximo-mésiale, dépourvu de nervures saillantes.

La mise en place de ces nervures permet l'extraction de lames issues du bord de la table convergeant dans leur partie distale vers la base cintrée du nucléus. Cette convergence s'effectue parfois d'une manière outrepassée, entretenant à la fois le cintrage mais aussi une légère carène en partie distale. Des nervures régulières, légèrement convergentes en partie distale, sont à présent installées sur la table permettant l'extraction en son centre d'enlèvements réguliers, rectilignes, plus courts que les enlèvements latéraux.

Ce débitage s'effectue dans un rythme de recul frontal, pour une exploitation volumétrique qui, du fait d'un cintre relativement étalé, est plutôt faciale. Cela favorise la production de lames relativement plus fines et plus larges que celles extraites de nucléus à exploitation volumétrique frontale évoqués précédemment.

Ces lames sont globalement rectilignes, parfois courbes en partie distale si elles parcourent toute la longueur de la table. Le tailleur doit doser précisément sa force pour extraire une lame qui ne file pas jusqu'au bout. Si le coup porté est trop faible, l'enlèvement est rebroussé. Le négatif de cet enlèvement est alors enlevé par l'extraction de lame plus épaisse et plus large, souvent outrepassée. Cet outrepassement permet la restauration d'une carène dista- 
le, compensant l'aplatissement du cintre causé par l'enlèvement plus large.

Un second plan de frappe, transversal par rapport au premier, peut être installé à des fins d'entretien. Les enlèvements qui en sont issus sont courts et ont pour but le recintrage de la partie distale du nucléus (compensant un cintre qui est par ailleurs plat dans sa partie proximomésiale).

2.2.3 - Une succession possible de ces deux modalités dans l'exploitation d'un même bloc

Plusieurs remontages effectués pour les silex jaspéroïdes $(n=5)$ révèlent la succession de ces deux modalités dans l'exploitation d'un même bloc. En effet, si un outrepasse ment survient lors de l'exploitation frontale d'un volume au cintre resserré, emportant le plan de frappe opposé, il est rédhibitoire pour la poursuite de cette modalité. Cependant, le volume résultant a parfois permis au tailleur de continuer le débitage suivant une modalité unipolaire convergente. Dans d'autres cas, lorsque le volume exploité suivant une modalité unipolaire convergente ne permet plus une exploitation faciale, le tailleur peut terminer la séquence par une exploitation frontale d'un volume réorienté à partir du petit coté du nucléus et initiée par l'installation d'un plan de frappe opposé.

2.2.4 - Une exploitation frontale d'un volume au cintre resserré unipolaire $(n=12)$

Une autre modalité exploite un volume au cintre polygonal ressemé de façon unipolaire. Un second plan de frappe est parfois installé de manière transversale au premier, permettant l'entretien du cintre. Dans la majorité des cas, la mise en forme est peu élaborée, le cortex étant le plus souvent conservé au niveau des flancs et du dos. Le plan de frappe présente fréquemment des traces d'aménagement. II s'agit d'une exploitation moins normalisée de blocs parfois de qualité médiocre. La productivité est alors limitée et l'abandon sera plus précoce.

\subsection{5 - La méthode du Raysse $(n=26)$}

D'après les travaux menés par L. Klaric sur les assemblages rayssiens, les burins du Raysse sont aujourd'hui considérés principalement comme des nucléus. Nous les considérons également comme tels aux Fieux, la présence d'une lamelle de la Picardie dans la couche $E$ étayant cette hypothèse. En parallèle de ce débitage lamellaire $(n=23)$, certains nucléus laminaires révèlent une exploitation proche de la méthode du Raysse, comme cela a été montré (Klaric 2003). Ils sont rares $(n=3)$ et seul l'un d'entre eux est clairement exploité selon cette modalité. Pour d'autres, l'aménagement du petit côté par une série d'enlèvements transversaux ne peut être qualifié de réel facettage. Ces indices rayssiens sont néanmoins complétés par la présence, certes anecdotique, de lames à facettage latéralisé oblique $(n=4)$. Nous n'avons pu, cependant, déteminer l'objectif de ce débitage.
2.2.6 - Un débitage sur tranche d'éclat : différents schémas opératoires lamellaires

La requalification des burins du Raysse en tant que nucléus nous amène à intégrer les burins plans, considérés comme des burins du Raysse atypiques, dans cette catégorie $(n=22)$, ainsi que certains burins multiples pouvant être qualifiés de burins du Raysse d'un côté et comportant des enlèvements plans d'un autre $(n=4)$.

D'autres éléments sont également susceptibles d'être des nucléus à lamelles : certains se rapprochent d'un débitage unipolaire très cintré témoignant d'un recul frontal avec un envahissement très peu prononcé des flancs $(n=9)$, deux autres, peu lisibles, sont bipolaires.

Tandis que les différentes formes de débitage sur bloc précédemment décrites témoignent d'une continuité lameslamelles dans la production, un débitage exclusivement lamellaire, réalisé sur tranche d'éclat, existe donc au sein de l'assemblage. Ce dernier concerne principalement des éléments se rapprochant plus ou moins précisément de la méthode du Raysse. D'autres schémas sont néanmoins présents auxquels pourraient être associés les burins carénés mentionnés précédemment (cf. § I.3.3).

\subsection{7 - Un débitage d'éclats $(n=9)$}

Plusieurs nucléus présentent, à leur stade d'abandon, des négatifs de petits éclats laminaires $(n=6)$, voire d'éclats $(n=3)$. Un remontage entre deux de ces éléments montre qu'il a pu y avoir une recherche de ce type de débitage. Le bloc de départ avait cependant été déjà exploité, probablement pour un débitage laminaire. De plus, aucun objectif relatif à cette production n'a été décelé au sein de l'outillage qui demeure donc anecdotique.

\subsection{8 - Une utilisation probable de la pierre tendre}

Nous n'avons pas quantifié les différents caractères observés concernant la préparation et le détachement des produits. Nous pouvons néanmoins noter que la majorité des produits présente un talon lisse, très souvent linéaire. Cette observation est à nuancer pour les lames de flanc qui présentent un talon souvent lisse plus épais. Une abrasion peut accompagner le détachement, mais elle n'est pas systématique ni très soignée.

Les parties proximales des lames (plus particulièrement en silex jaspéroïdes) portent de nombreux stigmates caractéristiques de l'utilisation de pierre tendre tels qu'ils ont pu être décrits : points d'impact prolongés par des bulbes marqués, présence de petites rides fines et serrées proches du point de contact, esquillement du bulbe (Pelegrin 2000). 


\begin{tabular}{|c|c|c|c|}
\hline Approvisionnement & Variétes de sillex & $\%$ de l'outillage & $\%$ d'artefacts bruts \\
\hline \multirow{2}{*}{ Local } & Jaspéroides de l'Infralias & 16,6 & 21,8 \\
\hline & Jurassique bajocien et bathonien & 8 & 31,1 \\
\hline \multirow{6}{*}{ Allochtone } & Sènonien & 35,5 & 20,9 \\
\hline & Campanien inférieur de type Belves & 1,5 & 0 , \\
\hline & Maastrichtien de Borgerac & 0.2 & 0 \\
\hline & Turonien inferieur de Fumel & 0,6 & 0,5 \\
\hline & Coniacien inférieur de Gavaudun & 0,4 & 0 \\
\hline & "Grain de Mil" & 0,9 & 0,4 \\
\hline \multirow[t]{2}{*}{ Local el allochlone } & Terliaire & 21,9 & 16,9 \\
\hline & Indéterminé & 14,4 & 8 \\
\hline
\end{tabular}

Tableau 4 - Les différentes matières premières caractérisées dans la couche $E$ des Fieux - les proportions en artéfacts bruts sont données pour un échantillon de 3600 pièces de plus de $1 \mathrm{~cm}$ (d'après l'étude de $P$. Chalard).

Table 4 - Various raw materials caracterized in the E layer of Les Fieux - raw artefacts ratio are given with a sample of 3600 items > 1cm (after P. Chalard's study).

\section{3- Un traitement différentiel des matières premières}

La caractérisation des différents types de silex a été réalisée par P. Chalard (tab. 4). Cela a permis de montrer, à travers l'étude typo-technologique, l'importance de l'aspect économique dans le traitement des matières siliceuses : des différences ont pu être observées à tous les stades de la chaîne opératoire.

\subsection{1 - Des objectifs orientés, d'autres polyvalents}

Le traitement différentiel des matières premières se révèle tout d'abord à travers les proportions d'outils (fig.15) :
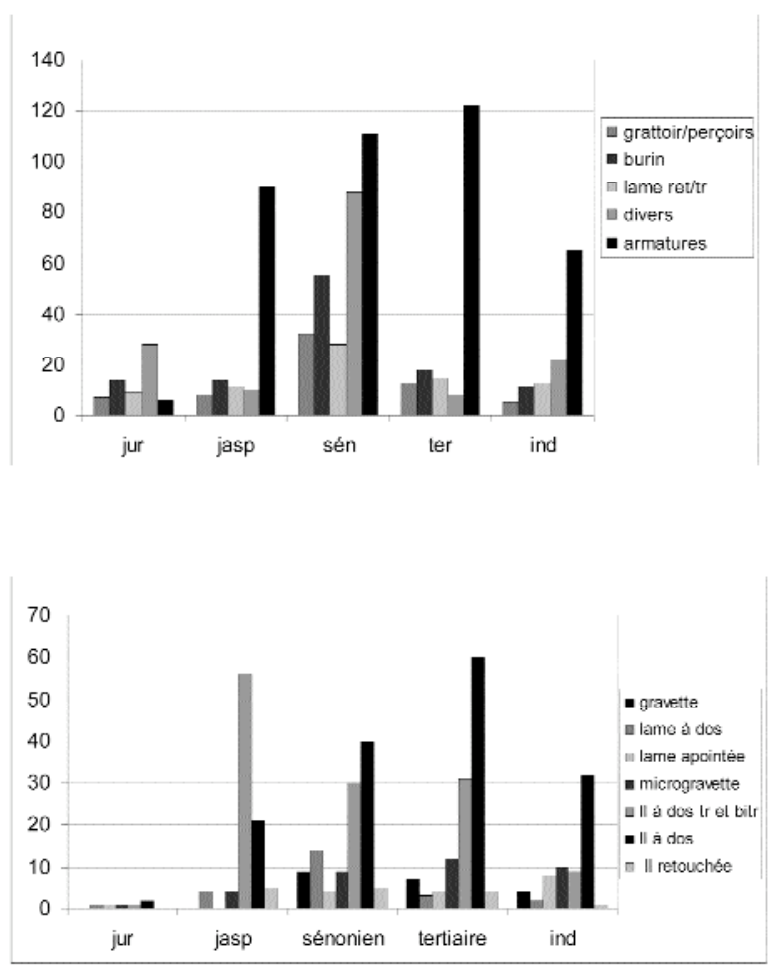

l'outillage à usage domestique en silex sénonien est en proportion plus importante que les armatures $(65 \%$ des outils) alors que les pourcentages sont inversés pour les silex jaspéroïdes et tertiaires. Par ailleurs, pratiquement aucune armature en silex du Jurassique n'est présente sur le gisement.

Ensuite, la répartition des divers types d'armatures par matière première montre une production très orientée des silex jaspéroïdes vers les lamelles à dos tronquées tandis que la production en silex sénonien est beaucoup plus variée, concernant l'ensemble des armatures (fig.15).
Figure 15 - En haut : répartition des types d'outils suivant les matières premières.

En bas : répartition des types d'armatures suivant les matières premières.

Figure 15 - Above : distribution of tool-types according to raw materials.

Below: distribution of projectile points types according to raw materials. 
2.3.2- Des modalités d'exploitation adaptées, d'autres marquées par la variabilité:

\subsubsection{1 - Les matériaux locaux}

\subsubsection{1 - Les Jaspéroïdes de l'Infralias}

L'orientation préférentielle dans la production en silex jaspéroïdes dévoile une exploitation adaptée à cette variété de silex.

En effet, les lamelles à dos tronquées sont des armatures que l'on peut envisager emmanchées latéralement, donc recherchées pour leurs qualités tranchantes. Or, le grain des silex jaspéroïdes est particulièrement fin, favorisant le tranchant des produits issus de cette matière.

En outre, la modalité opératoire majoritairement employée est unipolaire convergente. Cette modalité favorise la largeur et la finesse du support, accentuant son caractère tranchant. Par ailleurs, la finesse du grain, facilitant la propagation de l'onde de percussion, accentue les risques d'outrepassement des produits. Dans la mesure où ces phénomènes sont légers, ils ne perturbent pas toujours la poursuite d'un débitage intégrant ce type de produits, et peut-être même au contraire.

Ainsi, cette modalité, la plus employée pour les silex jaspéroïdes, est adaptée d'une part à une matière où il est nécessaire de gérer et d'anticiper le risque d'outrepasse ments, d'autre part à l'objectif d'une armature fine et tranchante.

Concemant le fractionnement de la chaîne opératoire, hormis les toutes premières phases, toute la séquence est présente sur le site jusqu'à l'abandon de l'outil.

\subsubsection{2 - Le Jurassique}

Les outils en silex jurassique sont essentiellement des outils à usage domestique. Cependant ces derniers sont peu élaborés (éclats retouchés, encoche, denticulé, burin sur cassure...) réalisés sur éclats, très souvent corticaux, issus des phases de mise en forme et d'entretien. Ces proportions ne sont pas révélatrices de l'exploitation effective de la matière la plus représentée parmi les restes abandonnés sur le site.

Cette exploitation est très différente de celle des jaspéroïdes. Tout d'abord, la matière a été introduite sur le site sous forme de rognons bruts, souvent de grand gabarit. Les modalités opératoires employées sont plus ou moins élaborées suivant la qualité des blocs au départ : ceux qui bénéficient d'une mise en forme révèlent une exploitation d'un volume au cintre polygonal resserré, mettant en jeu un ou deux plans de frappe suivant le degré d'élaboration possible. Ces modalités favorisent l'épaisseur et donc la robustesse du support, cette dernière étant déjà induite par une matière grenue. Enfin, les supports produits sont absents de l'outillage, ils ont donc été exportés, sous forme brute ou retouchée. Compte tenu de leur rectitude, de leur gabarit souvent laminaire et de leur robustesse, ils sont pour la plupart très différents des supports de lamelles à dos tronquées, se rapprochant de ceux des pointes de la Gravette.

\subsubsection{2 - Les matières allochtones}

L'exploitation des ressources allochtones a été détaillée en collaboration avec P. Chalard (Chalard et al. 2006). Le silex tertiaire, pouvant être issu d'un approvisionnement en partie local, a été exclu de nos considérations. Les matières allochtones sont alors regroupées dans un ensemble "Sénonien-Turonien". La polyvalence de ces matériaux, visible à travers la répartition des divers types d'outils, se retrouve dans leur traitement économique.

Tout d'abord, certaines spécificités sont à mettre en avant, notamment la quasi-exclusivité de ces matières comme support de production des burins du Raysse. Deux d'entre eux sont en Jurassique, les autres sont réalisés en Sénonien mais aussi dans un silex blanc porcelainé d'origine indéterminée. Notons cependant que ce n'est pas le cas des nucléus laminaires débités dans une méthode proche du Raysse qui sont également réalisés dans des matériaux locaux, jaspéroïdes et jurassiques.

Ensuite, les rares représentants d'un débitage d'éclats sont en silex sénonien. Cela témoigne d'une utilisation de supports variés en ces matières, en liaison avec la réalisation d'une large panoplie d'outils à usage domestique.

En effet, les supports d'outils à usage domestique sont intégrés dans la chaîne opératoire de production d'armatures par la récupération de sous-produits de formes diversifiées, même corticaux.

Les groupes ont alors choisi de transporter la matière sous forme variée, y compris brute. Cela permet, lors du débitage, la récupération sur place de sous-produits pour subvenir à différents types de besoins, traduisant ainsi une certaine économie du débitage (Perlès 1991).

2.3.3 - Des éléments pour une hypothèse d'homogénéité, premières données interprétatives.

La présente étude typo-techno-économique apporte des éléments de réflexion sur la question de l'homogénéité de la série. Formulons dans un premier temps, l'hypothèse où la couche $E$ correspondrait à un palimpseste d'occupations : il est alors possible d'envisager l'appartenance des lamelles à dos tronquées et du schéma unipolaire convergent qui leur est associé à un ensemble distinct du reste. Les acteurs de l' (ou des) occupation(s), relative(s) à cet ensemble, privilégieraient ainsi l'exploitation des silex jaspéroïdes au détriment de l'autre matière locale, le jurassique. D'autres occupants auraient alors eu une stratégie d'exploitation des ressources locales inverse, privilégiant pour leur part les silex du Jurassique.

Or, les remontages témoignent clairement de la succession possible de deux des principales modalités opératoires - unipolaire convergente et prismatique à deux plans de frappe - pour l'exploitation d'un même bloc, démontrant leur appartenance au même ensemble. Ainsi, les différents groupes peuvent utiliser des schémas opératoires similaires, privilégiant l'un ou l'autre. Sur quels critères pouvons-nous alors distinguer des ensembles différents au sein des silex allochtones ? En effet, ces derniers sont exploités suivant toutes les modalités et ont la particularité de représenter l'ensemble de l'outillage de la couche $\mathrm{E}$, contrairement aux silex locaux. Au final, aucun argument 
ne nous permet d'écarter les lamelles à dos tronquées du reste de l'ensemble.

Au contraire, il nous semble que le traitement différentiel des variétés de silex témoigne d'une complémentarité au sein d'une stratégie d'exploitation des ressources siliceuses, les regroupant dans le même ensemble archéologique.

II y a donc eu, selon notre interprétation, une exploitation adaptée des ressources locales au cours d'une occupation (ou de plusieurs occupations réalisées dans un temps relativement court par le même groupe). Ces matières, suivant leurs propriétés mécaniques, remplissent des objectifs différents dont l'usage peut être immédiat ou différé : les lamelles à dos tronquées produites sont abandonnées sur place tandis que les pointes de la Gravette sont vraisemblablement exportées.

Néanmoins, demeure le cas des éléments caractéristiques du faciès " rayssien ", qui ont toujours été en marge de cette étude. Ils sont beaucoup plus discrets et aucun raccord de débitage n'a permis de les mettre en relation avec les autres éléments gravettiens. En reprenant l'hypothèse d'un palimpseste, il est possible d'envisager l'existence d'un ensemble correspondant à un faciès rayssien "pur" tel qu'il a été défini par L. Klaric - dépourvu de pièces à dos et de schémas opératoires d'obtention de supports rectilignes (Klaric, 2003) - mélangé au reste de l'assemblage. Cependant, ces éléments " rayssiens " n'ont pas de localisation particulière, ils sont présents sur l'ensemble du niveau. De plus, les burins du Raysse sont réalisés dans des matières presque exclusivement allochtones. Les nucléus laminaires sont rares et débités selon une modalité, certes proche de la méthode du Raysse, mais qui semble mal maîtrisée. Au final, il est difficile d'isoler ces éléments pour reconstituer un niveau archéologique à part entière. Cela impliquerait par ailleurs le même cas de figure pour les autres niveaux gravettiens des Fieux qui présentent la même association. Par conséquent, il nous semble plus réaliste de considérer que l'ensemble de la couche $E$ est homogène.

\section{3 - LA COUCHE E AU SEIN DU TECHNOCOMPLEXE GRAVETTIEN : ÉLÉMENTS DE RÉFLEXION ET D'INTERPRÉTATION}

\section{1 - La couche $E$ : un exemple de stratégie d'acquisi- tion et d'exploitation des ressources siliceuses d'un groupe gravettien}

\subsection{1 - Une exploitation raisonnée des matières premières}

Résumons à présent les données que nous avons sur les différents types de matière première :

Les silex allochtones du Sénonien ont produit en grand nombre des outils à usage domestique. Ces derniers sont réalisés à partir des sous-produits de la chaîne opératoire de production d'amatures. Ainsi, les matières allochtones sont polyvalentes et peuvent être qualifiées de " matière première de voyage ", utilisées en réponse à l'ensemble des besoins que peut avoir le groupe durant ses déplacements, induisant un transport sous forme variée de la matière, du bloc brut à l'outil.

Ce stock diminuant en quantité mais aussi en volume, le groupe a exploité une matière locale - le Jurassique adaptée pour la production de supports laminaires relativement robustes afin de restaurer le stock de matière première, anticipant les besoins des futurs déplacements au sein du territoire.

Les silex jaspéroïdes ont fait l'objet d'une exploitation adaptée et orientée principalement vers la production de lamelles à dos tronquées. De plus, l'absence de fractionnement de la chaîne opératoire révèle un usage immédiat plus spécifique que pour les silex du Sénonien. Ce phénomène est révélateur d'un investissement technique fort dans la production d'armatures que l'on est tenté de mettre en relation avec l'activité de chasse pratiquée aux abords du site.

Ainsi, sans parler de réelle économie des matières premières, des préférences se manifestent dans l'exploitation des ressources siliceuses. Elles révèlent une adaptation du groupe aux opportunités offertes par les ressources locales et un investissement technique raisonné pour répondre à des objectifs présents ou futurs, complété par l'anticipation que constitue l'apport de matière première de voyage. Tout ceci donne une image d'un groupe maîtrisant son parcours au sein d'un territoire dont l'étendue et le mode d'exploitation restent à préciser.

3.1.2 - Réflexion sur la souplesse adaptative des Gravettiens

Le bilan effectué sur l'exploitation des ressources siliceuses de la couche $E$ illustre un modèle bien connu : “ [les stratégies économiques] peuvent être le résultat d'une cascade d'interactions entre trois types de contraintes: ressources du milieu, possibilités techniques au niveau des procédés et des méthodes, enfin besoins en produits finis" (Geneste 1991, p.16).

Les ressources du milieu ont été exploitées de manière adaptée à leurs propriétés. Ce phénomène dévoile un comportement gravettien relevant d'une certaine souplesse, ce qui va à l'encontre de l'image que l'on peut avoir d'un groupe qui se bornerait à transporter sur des kilomètres une matière première de qualité, leur permettant de mettre en œuvre un schéma opératoire pour une production normalisée. Les études récentes tendent à montrer que les groupes gravettiens peuvent utiliser des schémas opératoires variés dans leur production, possèdant une gamme élargie de possibilités techniques (Digan 2001 ; Klaric 2003 ; Lucas 2000 ; Pesesse 2003). L'ensemble de la couche $E$ illustre le lien pouvant exister entre la variabilité de certains schémas, les différentes matières présentes dans l'environnement et le statut d'une production anticipée ou à usage immédiat.

En développant l'idée de l'interaction entre les trois types de contraintes énoncées, la question de leur hiérarchie se pose : dans le cas des Fieux, est-ce parce qu'ils cher- 
chaient à produire des lamelles à dos tronquées que les paléolithiques ont ainsi exploité les silex jaspéroïdes ? Ou bien est-ce la présence de silex jaspéroïdes dans l'environnement de l'occupation qui a favorisé la production de ce type de produit, entraînant l'accroissement de la catégorie des lamelles à dos tronquées ?

Autrement dit, jusqu'où va la capacité d'adaptation du tailleur et par la même la souplesse des Gravettiens ? La diversité des armatures ne peut-elle pas être le résultat d'une recherche de combinaison optimale des trois types de contraintes pour répondre à l'objectif d'une occupation spécialisée?

Cela ne résout pas la question de l'association des éléments dits " rayssiens " aux amatures à dos et aux schémas producteurs de leurs supports. L'originalité de la couche $E$ soulève néanmoins la question de la fonction du site et des implications d'une éventuelle spécialisation dans la constitution des assemblages.

\subsection{3 - Conséquence sur l'interprétation des assemblages gravettiens}

Interpréter l'originalité de certains assemblages gravettiens du fait de la fonctionnalité de l'occupation a déjà été énoncé auparavant. Cependant, très peu ont entamé des démarches d'études concrètes étayant cette hypothèse (Rigaud 1978,1985).

Aujourd'hui la technologie lithique, associée à la pétroarchéologie, permet l'insertion de l'industrie lithique au sein d'une stratégie d'acquisition et d'exploitation des ressources minérales. Cette ouverture amène une vision plus globale de l'occupation du site, replacée au sein d'un territoire parcouru par le groupe. Des éléments de réponse à la question de la motivation de leurs déplacements et de l'éventuelle spécialisation des occupations pourront être avancés. Les hypothèses énoncées devront ensuite être testées par les études archéozoologiques. L'accumulation de ces démarches permettra de valider ou non l'hypothèse de la spécialisation des occupations comme l'un des facteurs du polymorphisme des assemblages gravettiens.

Les résultats de l'étude menée sur l'ensemble de la couche E apportent des éléments de réflexion sur les stratégies d'acquisition et d'exploitation des matériaux siliceux des groupes gravettiens. Parcourant des environnements différents, ils ont pu développer une souplesse adaptative leur permettant d'optimiser les opportunités offertes par les ressources de leur environnement proche, en fonction de leurs objectifs spécialisés ou non. Cette souplesse technique des groupes gravettiens, pouvant avoir un impact conséquent sur la constitution même de l'outillage, demande à être étudiée.

\section{2- Quelle attribution chrono-culturelle ?}

3.2.1- Reprise de l'hypothèse d'un palimpseste : conséquence sur l'attribution chrono-culturelle.

Même si l'étude typo-techno-économique étaye l'hypothèse d'homogénéité de l'ensemble de la couche $E$, envisageons une nouvelle fois le cas d'un palimpseste et voyons les attributions chrono-culturelles qui en découlent : les éléments " rayssiens " constitueraient un ensemble archéologique à part entière, mélangé à un ensemble gravettien à gravettes et lamelles à dos tronquées.

Ce demier, en l'absence de stratigraphie, peut être attribué au Gravettien ancien ou évolué, gardant dans les deux cas un caractère atypique par la présence de nombreuses lamelles à dos tronquées.

Certaines pointes de la Gravette portent une retouche de “ type Vachon " à l'extrémité basale $(n=6)$. II semble que cette demière affecte principalement les pointes du Gravettien moyen du Périgord et des Pyrénées ${ }^{2}$. Elle est néanmoins présente dans les couches ancienne (couche 5) et récente (couche 3) de l'Abri Pataud (Bricker 1995 ; Simonet A. 2005) et se trouve très bien représentée dans l'une des couches du Gravettien récent de l'Abri des Peyrugues (couche 22) (Allard 1994-1996). II est donc difficile, dans l'état actuel des recherches sur la structuration du Gravettien, d'interpréter d'une manière chronologique la présence de cette retouche sur les pointes de la Gravette de la couche E des Fieux.

L'importance des lamelles à dos tronquées n'a, jusqu'à présent, été mis en avant que dans l'industrie de la couche 2 de L'Abri Pataud. Cette caractéristique aurait tendance à rapprocher l'ensemble du Gravettien final (ex "Protomagdalénien "), mais ce faciès - défini principalement d'une part, par la présence de grandes lames retouchées, supports de burins majoritairement dièdres ou de grattoirs, d'autre part par l'absence (ou la rareté) des pointes et micropointes de la Gravette - ne correspond pas à la couche $E$ (Bordes F. 1978 ; Bordes F. et Sonneville-Bordes 1966 ; Bricker 1995 ; Clay 1968 ; Movius 1958 et 1968 ; SonnevilleBordes 1960 ; Guillermin en cours) ${ }^{3}$.

3.2.2 - Les données du sud-ouest de la France, hypothèses interprétatives

Par ailleurs, aucun ensemble " purement rayssien " (similaire à la Picardie) n'a été trouvé dans le Sud-Ouest de la France : les burins du Raysse sont toujours associés à des pièces à dos, à l'exemple de l'Abri Pataud (c.4), du Flageolet I (c.VII,VI et V) et de Solvieux (c. III.2 et M.6), (Bricker 1995 ; David 1973 ; Klaric 2003 ; Lucas 2000, 2002 ; Pottier 2005 ; Rigaud 1969 et 1982 ; Sackett 1999).

(2) Les pointes des Vachons sont présentes notamment dans la couche 4 de l'Abri Pataud ainsi que dans les couches gravettiennes de la grotte d'Isturitz (Simonet A . 2005 entre autres).

(3) Précisons par ailleurs que ces lamelles ne doivent pas être confondues avec les "éléments tronqués ", définis par D. de Sonneville-Bordes, qui sont des lames à dos tronqué au gabarit nettement plus large (Sonneville-Bordes 1960). Aucun indice chrono-culturd ne peut être extrait de ce phénomène caractérisant par ailleurs l'originalité de l'ensemble. 
En outre, cette coexistence est quasi-systématiquement associée à la présence de burins de Noailles ${ }^{4}$.

Aux Fieux, ces éléments sont absents mais si le burin du Raysse est un marqueur chrono-culturel fort, il nous permet d'attribuer l'occupation de la couche E des Fieux au Gravettien moyen à burins du Raysse.

Ainsi, la couche $\mathrm{E}$ des Fieux vient rejoindre les séries du sud-ouest de la France, questionnant les différentes hypothèses d'interprétation du faciès " rayssien".

L'étude de la couche 4 de l'Abri Pataud a amené $\mathrm{N}$. David, et par la suite $C$. Pottier, à définir un faciès noaillien, indépendant de la lignée gravettienne (David 1973 ; Pottier 2005). Pour N. David, le Noaillien inférieur, riche en burins de Noailles, évoluerait vers le Noaillien supérieur, riche en burins du Raysse. C. Pottier n'établit pas ces subdivisions, considérant une évolution continue, sans aucune rupture.

La couche $E$ contribue difficilement à étayer cette vision : d'une part elle ne possède aucun burin de Noailles, d'autrepart, la présence importante de pièces à dos ainsi que les schémas opératoires décrits rapprochent indubitablement la série de la famille gravettienne au sens large. En revanche, si nous nous référons au modèle maintenant le Rayssien dans une lignée évolutive gravettienne (Bosselin et Djindjian 1994), les burins du Raysse remplaçant les burins de Noailles, l'absence de ces derniers peut être interprétée comme un caractère évolué de la série. Notons cependant que l'explication des auteurs sur un changement motivé par une évolution technologique des burins est remise en question (Klaric 2003 ; Pottier 2005).

L. Klaric vient ébranler cette vision évolutive et avance une troisième hypothèse : celle de la considération du Rayssien comme une tradition technique distincte du reste de la lignée gravettienne, y compris du faciès moyen à burins de Noailles (Klaric 2003). II s'appuie sur les gisements de la Picardie et d'Arcy-sur-Cure, révélateurs d'une utilisation exclusive d'un système technique dit "rayssien ", profondément différent des systèmes gravettiens (plus anciens et plus récents) destinés à l'obtention de supports de pièces à dos. Ce modèle septentrional doit être testé par les ensembles du Sud-Ouest. En effet, ces derniers, parmi lesquels nous intégrons à présent la couche $\mathrm{E}$ des Fieux, révèlent l'association de ces deux systèmes.

Si nous poursuivons l'idée de L. Klaric, il est alors possible d'envisager l'existence de groupes différents porteurs de ces traditions techniques. Auquel cas, pourquoi ne pas avancer l'hypothèse selon laquelle cette coexistence est le témoignage de liens entre un groupe gravettien méridional avec un groupe "rayssien " plus septentrional ? Cela s'accorderait bien avec la quasi-exclusivité de l'emploi de matériaux allochtones pour la confection des burins du Raysse ainsi que la rareté et la maladresse dans l'emploi de la modalité laminaire qui leur est associée.
Cette hypothèse appuierait la thèse de la contemporanéité de deux groupes, souvent décrits comme noaillien et rayssien. II est néanmoins difficile de rattacher la couche E au premier en l'absence de burins de Noailles, même si ce caractère peut être attribué à l'éventuelle spécialisation de l'occupation. II est alors possible d'envisager la perduration de ce phénomène de contemporanéité dans les faciès récents.

Nous ne pouvons conclure sur ces différentes interprétations. Une séquence stratigraphique plus septentrionale, apporterait par ailleurs des éléments déterminants dans ces considérations. Les études doivent être multipliées, axées notamment vers la pétroarchéologie.

\section{4 - CONCLUSION}

L'étude de la couche E des Fieux met en valeur plusieurs caractères problématiques inhérents aux assemblages gravettiens. Les premières étapes de son étude montrent les dangers que peut comporter une interprétation chronoclimatique du remplissage des gisements.

Ensuite, l'originalité de la série pose des questions déjà soulevées par différents préhistoriens confrontés à l'interprétation du polymorphisme des assemblages gravettiens, notamment de l'impact de la fonctionnalité du site sur la constitution des assemblages. La technologie lithique replace l'ensemble lithique au sein d'une stratégie économique d'exploitation des matières premières, induisant une réflexion sur la mobilité du groupe (Chalard et al. 2006). Ces éléments ne pourront réellement donner corps à une modélisation de l'exploitation et de l'occupation d'un territoire que confrontés aux données d'autres spécialités telles que l'archéozoologie.

Néanmoins, les éléments de stratégies économiques décelés aux Fieux révèlent une véritable souplesse adaptative d'un groupe gravettien optimisant les opportunités offertes par leur environnement en fonction de leurs objectifs, pouvant peser sur la variabilité des assemblages. Cette adaptation peut alors mettre en valeur différentes facettes de leur système technique en fonction des étapes du parcours du teritoire. De nouveaux critères de comparaisons de gisements, replacés dans leur contexte, peuvent ainsi être définis.

Enfin, concernant la dimension culturelle d'un faciès gravettien, l'ensemble de la couche $E$ amène également des éléments de réflexion sur l'interprétation de la présence de burins du Raysse dans les séries du sud-ouest de la France. Ces derniers étaient jusqu'à présent considérés comme des marqueurs forts du Gravettien moyen mais les travaux récents (Klaric 2003), remettent en question cette vision chronologique, introduisant une dimension géographique. II décrit ainsi un faciès septentrional " purement"

(4) Deux gisements présentent l'association de burins du Raysse et de gravettes sans burins de Noailles : les Jambes et les Artigaux (Célérier 1969, 1967 ; Lenoir 1977). Cependant ces données doivent être considérées avec une certaine réserve : la stratigraphie du premier gisement n'a pas fait l'objet d'une étude taphonomique critique et la série du deuxième est issue d'un ramassage de surface. 
rayssien, dépourvu du système de production des armatures à dos. L'association des deux systèmes dans les séries du sud-ouest de la France demande à être interprétée. Ce phénomène peut notamment être envisagé comme le témoignage de contacts et d'échanges entre des groupes contemporains, détenteurs de traditions techniques différentes. Quoi qu'il en soit, d'après les stratigraphies du Sud-Ouest et dans l'attente de nouvelles données, l'ensemble de la couche $E$ pourrait être attribué au Gravettien moyen, mais l'absence de burins de Noailles plaide en faveur d'un caractère plus évolué de la couche $E$, la rapprochant du Gravettien récent.

Ainsi, les trois prismes d'interprétation du polymorphisme du technocomplexe gravettien sont toujours d'actualité, regroupant la chronologie, le régionalisme et la fonctionnalité des sites.

\section{Remerciements}

Nous remercions tout d'abord F. Bon pour son encadrement ayant permis la réalisation de ce travail, L. Klaric pour sa relecture, M. Allard, P. Chalard et M. Jarry pour leur collaboration au sein de l'ACR Quercy, ainsi qu'A. Morala pour son écoute et ses remarques.

\section{BIBLIOGRAPHIE}

ALLARD M. 1994-1996 - Rapport de fouilles. S.R.A MidiPyrénées.

ALLARD M., DRIEUX M., JARRY M., POMIES M.P. et RODIERE J. 1997 - Perles en bois du gisement des Peyrugues, à Orniac (Lot); Hypothèse sur l'origine du Protomagdalénien.Paléo, t.9, p.355-369.

BELOUNIS N. 1987 - Contribution à la climatologie et à la chronologie du Paléolithique en Haut-Quercy. Les gisements en grotte des Fieux et des Graves. Thèse de 3ème cycle, Université de Bordeaux I, 240 p., 112 fig. 1 tab.

BON F. et BORDES J-G. 2005 - L'Aurignacien des Fieux (Miers, Lot). In (Jarry M., coord.) : Cultures et environnements paléolithiques : mobilités et gestions des teritoires des chasseurs-cueilleurs en Quercy. Rapport d'activités 2005 de l'Action Collective de Recherche sur le Quercy, 8 p., 1 fig.

BORDES F. 1968 - La question Périgordienne, La Préhistoire, problèmes et tendances, Editions du CNRS, p.59-70, 3 fig.

BORDES F. 1978 - Le Protomagdalénien de Laugerie-Haute Est (fouilles F.Bordes). Bulletin de la Société Préhistorique française, t.75 (11-12), p.501-521, 11 fig. 2 tab.

BORDES F. et LABROT J. 1967 - La stratigraphie du gisement de Roc-de-Combe (Lot) et ses implications. Bulletin de la Société Préhistorique française, t.64 (1), p.15-28, 6 fig.
BORDES F. et SONNEVILLE-BORDES D.de 1966 Protomagdalénien ou Périgordien VII? L'Anthropologie, t.70 (1-2), p.113-122, 5 fig.

BOSSELIN B. et DJINDJIAN F. 1994 - La chronologie du Gravettien français. La Préhistoire européenne, t.6, p.77-115.

BRICKER H.M. 1995 - Le paléolithique supérieur de l'abri Pataud (Les Eyzies, Dordogne). Les fouilles de H.L. Movius $J r$, Documents d'Archéologie Française, Maison des Sciences de l'Homme, $328 \mathrm{p}$.

CAHEN D., KARLIN C., KEELEY H.D. et VAN NOTEN F. 1980 - Méthodes d'analyse technique, spatiales et fonctionnelles d'ensembles lithiques. Helinium, t.XX, p.209-259.

CÉLÉRIER G. 1967 - Le gisement périgordien supérieur des «Jambes», commune de Périgueux (Dordogne). Bulletin de la Société Préhistorique française, t.64, Etudes et travaux $n^{\circ} 1$, p.53-68, 4 fig. 1 tab.

CHADELLE J.-P., GENESTE J.-M. et PLISSON H. 1990 Processus fonctionnels de formation des assemblages technologiques dans les sites du Paléolithique supérieur. Les pointes de projectiles lithiques du Solutréen de la gro tte de Combe-Saunière (Dordogne, France). In : 25 ans d'études technologiques, Actes des Xlème rencontres nationales d'Archéologie et d'Histoire d'Antibes, 1990 APDCA, p. 275-288.

CHALARD P., GUILLERMIN P. ET JARRY M. 2006 Acquisition et exploitation des silex allochtones au Gravettien : l'exemple de la couche E des Fieux. In [Bressy C., Burke A., Chalard P. et Martin H. dir.]. Notions de territoire et de mobilité. Exemples de l'Europe et des premières nations en Amérique du Nord avant le contact européen. Actes de sessions présentées au $X^{e}$ congrès annuel de l'Association Européenne des Archéologues (E.A.A.), Lyon, 8-11 septembre 2004, Liège, ERAUL 116, 2006, p. 29-40, 10 fig., 2 tab.

CHAMPAGNE F. 1977 - Essai d'interprétation chronologique des niveaux supérieurs de la grotte des Fieux, à Miers, Lot. Pré-publication du colloque de Bordeaux, Compléments.1977 16 p., 5 fig.

CHAMPAGNE F. 1986-1987 - Aurignacien et Périgordien dans le Haut-Quercy: l'apport des Fieux. Antiquités Nationales, t.18-19, p.63-68, 1 tab.

CHAMPAGNE F. ET JAUBERT J. 1979 - La grotte des Fieux à Miers (Lot). Bilan de 13 années de recherche. XXI ème Congrès préhistorique de France, Montauban-Cahors 1979, Vol. 1, p. 85-104, 13 fig.

CHAMPAGNE F., CHAMPAGNE C., JAUZON P. et NOVEL PH. 1990 - Le site des Fieux. Gallia Préhistoire, t.32 (1), p.1-28.

CHAMPAGNE F., CHAMPAGNE DR C. et NOVEL PH. 1996 Le site des Fieux dans les causses du Quercy. Relations entrel'homme préhistorique et son milieu. La vie préhistorique, Société Préhistorique Française. p.150-155. 
CHAMPAGNE F. ET JAUBERT J. 1986 - Un exemple de remplissage archéologique en milieu karstique: la grotte des Fieux, à Miers (Lot). Bulletin de la Société Méridionale de Spéléologie et Préhistoire, t.XXVI, p.21-33, 3 fig.

CLAY R.B. 1968 - The protomagdalénian culture. Unpublished Ph.D., dissertation presented to the Dept. of Anthropology, Southern Illinois University, 2 vol., 660 p., 82 fig. 198 tab.

QOOTTES J., LORBLANCHET M., FAU M.F. et PEYRE G. 1990 - L'abri périgordien de la Bergerie, à Saint-Géry (Lot). Bulletin de la Société Préhistorique française, t.87 (10-12), p.342-357, 15 fig. 3 tab.

COULONGES L. 1949 - Le gisement paléolithique de Cavart, Lot, commune de Montcabrier. L'Anthropologie, t.53, p.558-560, 2 fig.

DAVID N.C. 1973 - On upper paleolithic society, ecology and technological change: the Noaillian case. The explanation of culture change. p.277-303.

DIGAN M. 2001 - Le gisement de la Vigne Brun (Loire): première étude de l'industrie lithique de l'unité KL19. Thèse de doctorat de Préhistoire de l'Université de Lille I. 2 vol. 342 p.

GENESTE J-M. 1991 - Systèmes techniques de production lithique: variations techno-économiques dans les processus de réalisation des outillages paléolithiques. Techniques et cultures, t.17-18, p.1-35, 5 fig.

GLORY A. 1965 - Nouvelles découvertes de dessins nupestres sur le Causse de Gramat (Lot). Bulletin de la Société Préhistorique française, t.62, Etudes et travaux $\mathrm{n}^{\circ} 3$, p.528-538, 10 fig.

GUILLERMIN P. 2004 - Réflexions sur l'interprétation des industries gravettiennes à partir de l'étude techno-typologique d'une occupation spécialisée: la couche $E$ du gisement des Fieux (Miers, Lot). Mémoire de DEA de l'Université de Toulouse Le Mirail. 99 p. 48 fig. 3 tab.

GUILLERMIN P. 2005 - Les Périgordiens étaient-ils aussi quercinois ? In (Jarry M., coord.) : Cultures et environne ments paléolithiques : mobilités et gestions des territoires des chasseurs-cueilleurs en Quercy. Rapport d'activités 2005 de l'Action Collective de Recherche sur le Quercy. 28 p. 20 fig.

JAUBERT J. 1979 - L'Epipaléolithique du Haut-Quercy. Maîtrise de Second Cycle, Université de Paris I. 209 p.

KLARIC L. 2003 - L'unité technique des industries à burins du Raysse dans leur contexte diachronique. Réflexions sur la diversité culturelle au Gravettien à partir des données de la Picardie, d'Arcy-sur-Cure, de Brassempouy et du Cirque de la Patrie. Thèse de Doctorat de l'Université de Paris I, 429 p.
KLARIC L., AUBRY T. et WALTER B. 2002 - Un nouveau type d'armature en contexte gravettien et son mode de production sur les burins du Raysse (la Picardie, commune de Preuilly-sur-Claise). Bulletin de la Société Préhistorique française, t.99 (4), p.751-764.

LAVILLE H. et RIGAUD J.-PH. 1973 - The perigordain V industries in Perigord: typological variations, stratigraphy and relative chronology. World Archaeology, t.4 (3), p.330338, 2 fig. 2 tab.

LENOIR M. 1977 - Un gisement de plein air du Périgordien supérieur en Gironde: les Artigaux à Camiacet Saint-Denis. Bulletin de la Société Préhistorique française, t.74, Etudes et Travaux n`2, p.518-530.

LE TENSORER J.-M. 1981 - Le Paléolithique en Agenais, Cahiers du Quatemaire, 3, p.526. 212, 55 fig.

LORBLANCHET M. 1984a - L'art des cavernes. Atlas des grottes ornées paléolithiques françaises. Maison de la Culture, Imprimerie nationale, Paris.

LORBLANCHET M. 1984b - Nouvelles découvertes d'art pariétal en Quercy. L'art pariétal paléolithique, Périgueux. Le Thot, Ministère de la culture, p. 79-105.

LUCAS G. 2000 - Les industries lithiques du Flageolet (Dordogne) approche économique, technologique, fonctionnelle et spatiale. Thèse de doctorat de Préhistoire et Géologie du Quatemaire de l'Université de Bordeaux I, 2 vol, $600 \mathrm{p}$.

LUCAS G. 2002 - A propos des burins du Raysse du Flageolet I (Dordogne, France). Paléo, t.14, p.63-76.

MORALA A. 1984 - Périgorden et Aurignacien en HautAgenais. Etudes d'ensembles lithiques. Archives d'Ecologie Préhistorique, Ecole des Hautes Etudes en Sciences Sociales, Toulouse, Mémoire 7, 140 p., 42 fig.

MOVIUS H.L. Jr. 1958 - The Proto-Magdalenien of the Abri Pataud, les Eyzies (Dordogne). V internationalen kongress für Vor-und Frühgeschichte, Hambourg, 1958, p. 561-565.

MOVIUS H.L. Jr. 1968 - Segmented Backed Bladelets. Quärtar, t.19, p.239-249.

NOUGIER L-R. et BARRIERE C. 1965a - La grotte omée des Fieux (Commune de Miers, Lot). Annales de la Faculté des Lettres et Sciences Humaines de Toulouse, t. I.5, p.115-134, 18 fig.

NOUGIER L-R. et BARRIERE C. 1965b - La nouvelle grotte préhistorique des Fieux et ses mains négatives. $\mathrm{La}$ Nature, t.3359, p.81-86.

O'FARRELL M. 1996 - Approche technologique te fonctionnelle des pointes de la Gravette, une analyse archéologique et expérimentale appliquée à la collection de Corbiac (Dordogne) Mémoire de DEA de l'Université de Bordeaux I, 111 p. 
O'FARRELL M. 2000 - Les pointes de la Gravette de Corbiac (Dordogne) et considérations sur la chasse au Paléolithique supérieur ancien. Approches fonctionnelles en Préhistoire. XXV ème Congrès Préhistorique de France, 2000, 121-138p., 7 fig. 2 tab.

PERLES C. 1991 - Economie des matières premières et économie du débitage: deux conceptions opposées? Xlème rencontre internationale d'Archéologie d'Antibes. 25 ans d'études technologiques en Préhistoire: bilan et perspectives. Actes des rencontres 18-19-20 octobre 1990, Juan-les-Pins:APDCA, 1991, p. 35-45.

PESESSE D. 2003 - Approche du comportement technique au Gravettien: l'industrie lithique de l'unité OP 10 de la Vigne Brun, Université de Provence, 192 p.

PEYRE G. 1988 - L'Abri de la Bergerie, à Saint-Géry (Lot). Toulouse, Ecole des Hautes Etudes en Sciences Sociales, 1988, 127 p., 61 fig.

POTTIER C. 2005 - Le Gravettien moyen de l'abri Pataud (Dordogne, France): le niveau 4 et l'éboulis 3/4. Etude technologique et typologique de l'industrie lithique. Mémoire de doctorat du Museum d'Histoire Naturelle, 396 p.

RENAULT-MISKOVSKY J. 1983 - Evolution de la végétation et des climats durant les derniers 20000 ans du Paléolithique supérieur dans le Sud-Est de la France. Rivista di Scienze Preistoriche, t.XXXVIII, 1-2, p.117-121, 1 tab.

RIGAUD J.-PH. 1969 - Note préliminaire sur la stratigraphie du gisement du Flageolet I à Bézenac (Dordogne). Bulletin de la Société Préhistorique française, t.66 (3), p.73-75. 2 fig.

RIGAUD J.-PH. 1976 - Les civilisations du Paléolithique supérieur en Périgord. La Préhistoire française, t.l, vol.2, p.1257-1270, 2 fig.

RIGAUD J.-PH. 1978 - The significance of variability amoung lithic artefacts: a specific case from southwestern France. Journal of Anthropological research, t.34, n³, p.299-310.
RIGAUD J.-PH. 1982 - Le Paléolithique en Périgord: les données du Sud-Ouest Sarladais et leurs implications. Thèse de doctorat d'état de l'Université de Bordeaux I, 2 vol., 242 p., 294 fig. 17 tab.

RIGAUD J-PH. 1985 - Réflexions sur la signification de la variabilité des industries lithiques paléolithiques. Colloque "La signification culturelle des industries lithiques», Liège, 1985, Vol. 239, BAR International, p. 374-380.

SACKETT J. 1999 - The archaeology of Solvieux: an upper open air site in France. Monumenta Archaeologia, Institute of Archaeology, University of California, 19.

SIMONET A. 2005 - Les amatures lithiques. Méthodes d'étude et enjeux : l'exemple des armatures gravettiennes d'Isturitz. Mémoire de DEA de l'Université de Toulouse Le Mirail. 94 p. 62 fig.

SONNEVILLE-BORDES D. de 1960 - Le Paléolithique Supérieur en Périgord. 2 vol., 558 p., 295 fig. 64 tab.

SONNEVILLE-BORDES D. de, 2002 - Les industries de Roc-de-Combe (Lot). Périgordien et Aurignacien. Bulletin de Préhistoire du Sud-Ouest, t.9, p.121-161, 29 fig. 2 tab.

SONNEVILLE-BORDES D. de et PERROT J. 1954 Lexique typologique du Paléolithique Supérieur. Bulletin de la Société Préhistorique française, t.51 (7), p.327-335, 4 fig.

SONNEVILLE-BORDES D. de et PERROT J. 1955 Lexique typologique du Paléolithique Supérieur. Bulletin de la Société Préhistorique française, t.52 (2), p.76-79, 2 fig.

SONNEVILLE-BORDES D. de et PERROT J. 1956 Lexique typologique du Paléolithique Supérieur. Bulletin de la Société Préhistorique française, t.53 (8) et 53 (9), p.408412 et 547-549, 3 et 5 fig. 